\title{
Research Article Wavelet Transform for Processing Power Quality Disturbances
}

\author{
S. Chen and H. Y. Zhu \\ School of Electrical and Electronic Engineering, Nanyang Technological University, 50 Nanyang Avenue, Singapore 639798
}

Received 29 April 2006; Revised 25 January 2007; Accepted 17 February 2007

Recommended by Irene Y. H. Gu

\begin{abstract}
The emergence of power quality as a topical issue in power systems in the 1990s largely coincides with the huge advancements achieved in the computing technology and information theory. This unsurprisingly has spurred the development of more sophisticated instruments for measuring power quality disturbances and the use of new methods in processing and analyzing the measurements. Fourier theory was the core of many traditional techniques and it is still widely used today. However, it is increasingly being replaced by newer approaches notably wavelet transform and especially in the post-event processing of the time-varying phenomena. This paper reviews the use of wavelet transform approach in processing power quality data. The strengths, limitations, and challenges in employing the methods are discussed with consideration of the needs and expectations when analyzing power quality disturbances. Several examples are given and discussions are made on the various design issues and considerations, which would be useful to those contemplating adopting wavelet transform in power quality applications. A new approach of combining wavelet transform and rank correlation is introduced as an alternative method for identifying capacitor-switching transients.
\end{abstract}

Copyright (c) 2007 S. Chen and H. Y. Zhu. This is an open access article distributed under the Creative Commons Attribution License, which permits unrestricted use, distribution, and reproduction in any medium, provided the original work is properly cited.

\section{INTRODUCTION}

Power quality is an umbrella terminology covering a multitude of voltage disturbances and distortions in power systems $[1,2]$. It is often taken as synonymous to voltage quality as electrical equipment is generally designed to operate on voltage supply of certain "quality." However, "quality" is a subjective matter as it depends very much on the individual requirements and circumstances. Voltage that is considered good for operating water heater may not be adequate for powering computers. In essence, power quality is a compatibility issue between the supply systems and loads [3]. As long as both can coexist without causing any ill effects on each other, the quality can be regarded as good or adequate. Hence, the scope of power quality is often extended to include imperfections in the design of supply system such as unbalanced transmission/distribution lines, poor connections, and inapt groundings.

Nonetheless, the majority of disruptions recognized as power quality problems involve electromagnetic phenomena that cause the supply voltage to deviate from its ideal characteristics of constant frequency $(50 / 60 \mathrm{~Hz})$, constant voltage magnitude (nominal values), and completely sinusoidal [1]. These phenomena can be divided into two broad cat- egories of time-varying and steady-state (or intermittent) events. The former group comprises voltage transients, dips, swells, and interruptions. They normally occur for a brief period of time (several milliseconds), but are often severe enough to cause wide-ranging disruptions to many electrical loads. Voltage dips lasting 5-6 cycles are known to cause programmable logic controller (PLC) in factories to malfunction. The latter group includes voltage unbalances, harmonic and interharmonic distortions, voltage fluctuation, notching, and noise. These steady-state (or semi-steady-state) phenomena would act subtly over a certain period of time before disruption occurs or intolerable condition surfaces. Harmonic voltage causes additional stress on equipment insulation, shortening their useful life. The eventual insulation breakdown often occurs after the equipment is being subjected to the distortion over extended period of time.

Signal processing is generally called upon when there is a need to extract specific information from the raw data, which typically in power systems are the voltage and current waveforms. The objectives of collecting data through measurements or simulations largely dictate which signal processing technique is to be utilized [4]. In power quality context, an evaluation often involves several phases that can be broadly divided into problem identification, classification 
and characterisation, followed by solution assessment and design. Further processing may be necessary if the results are to be presented in some special way. Designer of power conditioner would need to know the worst-case disturbance/distortion levels with much detailed, and both the magnitude and phase angle are equally important for the conditioner operation. On the other hand, a facility manager evaluating the overall quality level would prefer an overview of the measurements incorporating some statistical summaries. In such cases, magnitude would probably be sufficient. Regulator monitoring customers' compliance to limits would need the data processed and the results presented according to the methods stipulated in the regulation, standard or contract. Although one can argue that similar techniques can be applied in all scenarios, but the degree of processing or summarization is often different, largely affected by the length of the evaluation period.

With the advancement in measurement technology, an increasing volume of data is being gathered and it needs to be analyzed. It is highly desirable if the analysis is automated. Signal processing is therefore called upon for identification, classification and characterization. The techniques used vary, depending on the characteristics of the phenomena. As power systems use AC (alternating current), the RMS (root-mean-square) quantity is the most commonly used measure for voltage magnitude. Although it is meant for periodic waveform, it is often taken as a rough estimate of the nonperiodic or time-varying voltage variations. Voltage dips, swells, and interruptions are often characterized and classified using this quantity. When more explicit information is needed, such as evaluating disturbance propagation, time-frequency decomposition methods are necessary. Discrete Fourier transform (DFT) is a convenient way of visualizing stationary and periodic signal from its frequency content viewpoint. It is also applied to nonstationary signals but with added windowing to focus on certain period of time. This is called short time Fourier transform (STFT), allowing some tracing of the magnitude variations. Harmonic distortions are typically handled in this manner but the constraint placed on the frequency resolution makes it difficult to extend STFT to the analysis of interharmonics.

Fast voltage transients require the peak magnitudes and rise times to be determined accurately. For oscillatory transient, its predominant frequency needs to be derived before computing its magnitude. DFT is often used even though these waveforms are not periodic and last for less than one fundamental cycle since it is often necessary to determine their spectra content. Estimation techniques such as Kalman filtering are also called upon when there are uncertainties in the data. For other analyses that consider the effect of sensitive loads such as flickering of incandescent lamp due to voltage fluctuation, the data processing needs to mimic the behavior of lamp responses, human visual and psychological perception. Finally, after identification, classification, and characterization, the relevant information needs to be stored for future reference. Although the signal processing undertaken in these steps can be taken as some form of compression, further processing and threshold op- eration is often carried out to reduce the amount of data stored.

Wavelet transform (WT) is increasingly being proposed for the above processing in place of Fourier-based techniques. The primary reason for advocating WT is that it does not need to assume that the signal is stationary or periodic, not even within the analysis window. This makes it highly suitable for tracing changes in signal including fast changes in high-frequency components. WT is a time-scale decomposition technique and is generalized as a form of time"frequency band" analysis method. It not only traces signal changes across the time plane, but it also breaks signal up across the frequency plane. In discrete wavelet transform (DWT), signal is broken into multiple frequency bands, instead of a discrete number of frequency components as in DFT. With this character, WT is more appropriate if one is unsure of the exact frequency. Fortunately, most analyses do not require the exact frequency since a lumped quantity (frequency band) is sufficient to achieve their purposes. However, with power system engineering heavily entrenched in Fourier's techniques, it remains questionable if wavelet techniques are applicable and useful for the representation and analysis of voltage disturbances encountered in power systems [5].

This paper reviews the wavelet transform as a signal processing tool for processing power-quality-related disturbance waveforms. Section 2 provides a succinct introduction of WT and dwells into the properties of its basis functions. It explains the flexibilities and options inherent in the WT procedure, and demonstrates how they can be employed in power quality analysis. The challenges as well as opportunities presented by this new signal processing technique are traded side-by-side with respect to the requirements in analyzing power quality data. In Section 3, some exemplary uses of WT in power quality studies are presented. This is followed by Section 4 detailing various important factors that must be considered when contemplating wavelet approach in power quality applications. Section 5 describes a new approach of combining rank correlation with WT for identifying the capacitor-switching event. The conclusions and recommendations are given on which power quality phenomena WT is suitable for use and vice versa.

\section{WAVELET ANALYSIS}

Wavelet analysis is a technique for carving up function or data into multiple components corresponding to different frequency bands. This allows one to study each component separately. The main idea existed since the early 1800 s when Joseph Fourier first discovered that signals could be represented as superposed sine and cosine waves, forming the basis for the infamous Fourier analysis. From the beginning of 1990s, it began to be utilized in science and engineering, and has been known to be particularly useful for analyzing signals that can be described as aperiodic, noisy, intermittent, or transient [6]. With these traits, it is widely used in many applications including data compression, earthquake prediction, and mathematical applications such as computing 
numerical solutions for partial differential equations [7]. In recent years, it is increasingly being used in many power system applications including power quality measurement and assessment [8].

Wavelet analysis is a form of time-frequency technique as it evaluates signal simultaneously in the time and frequency domains. It uses wavelets, "small waves," which are functions with limited energy and zero average,

$$
\int_{-\infty}^{+\infty} \psi(t) d t=0
$$

The functions are typically normalized, $\|\psi\|=1$ and centered in the neighborhood of $t=0$. It plays the same role as the sine and cosine functions in the Fourier analysis. In wavelet transform, a specific wavelet is first selected as the basis function commonly referred to as the mother wavelet. Dilated (stretched) and translated (shifted in time) versions of the mother wavelet are then generated. Dilation is denoted by the scale parameter $a$ while time translation is adjusted through $b$ [9],

$$
\psi_{a, b}(t)=\frac{1}{\sqrt{a}} \psi\left(\frac{t-b}{a}\right),
$$

where $a$ is a positive real number and $b$ is a real number. The wavelet transform of a signal $f(t)$ at a scale $a$ and time translation $b$ is the dot product of the signal $f(t)$ and the particular version of the mother wavelet, $\psi_{a, b}(t)$. It is computed by circular convolution of the signal with the wavelet function

$$
\mathcal{W}\{f(a, b)\}=\left\langle f, \psi_{a, b}\right\rangle=\int_{-\infty}^{+\infty} f(t) \cdot \frac{1}{\sqrt{a}} \psi^{*}\left(\frac{t-b}{a}\right) d t .
$$

A contracted version of the mother wavelet would correspond to high frequency and is typically used in temporal analysis of signals, while a dilated version corresponds to low frequency and is used for frequency analysis.

With wavelet functions, only information of scale $a<1$ corresponding to high frequencies is obtained. In order to obtain the low-frequency information necessary for full representation of the original signal $f(t)$, it is necessary to determine the wavelet coefficients for scale $a>1$. This is achieved by introducing a scaling function $\phi(t)$ which is an aggregation of the mother wavelets $\psi(t)$ at scales greater than 1 . The scaling function can also be scaled and translated as the wavelet function,

$$
\phi_{a, b}(t)=\frac{1}{\sqrt{a}} \phi\left(\frac{t-b}{a}\right) .
$$

With scaling function, the low-frequency approximation of $f(t)$ at a scale $a$ is the dot product of the signal and the particular scaling function [9], and can be computed by circular convolution

$$
\mathcal{L}\{f(a, b)\}=\left\langle f, \phi_{a, b}\right\rangle=\int_{-\infty}^{+\infty} f(t) \frac{1}{\sqrt{a}} \phi^{*}\left(\frac{t-b}{a}\right) d t .
$$

Implementation of these two transforms (3) and (5) can be done smoothly in continuous wavelet transform (CWT) or discretely in discrete wavelet transform (DWT). The details are described in Appendix A.

\subsection{Multiresolution analysis}

One important trait of wavelet transform is that its nonuniform time and frequency spreads across the frequency plane. They vary with scale $a$ but in the opposite manner, with the time spread being directly proportional to $a$ while frequency spread to $1 / a$. This effect is best illustrated by the time-frequency boxes as shown in Figure 1 for short time Fourier transform (STFT) and DWT. In STFT, the time and frequency resolutions ( $\Delta t$ and $\Delta f$ ) are constant as illustrated by the fixed square boxes over the time-frequency plane. On the other hand, the resolutions of DWT vary across the planes. At low frequency when the variation is slow, the time resolution is coarse while the frequency resolution is fine. This enables accurate tracking of the frequency while allowing sufficient time for the slow variation to transpire before analysis. On the contrary, in the high-frequency range, it is important to pinpoint when the fast changes occur. The time resolution is therefore small, but the frequency resolution is compromised. However, it is generally not necessary to know the exact frequency in this range.

It is to be noted that as the resolutions vary, the area of the time-frequency boxes remain unchanged. This area is lower-bound by a limit as stipulated by the Heisenberg uncertainty principle "the more precisely the position is determined, the less precisely the momentum is known in this instant and vice versa." This principle asserts that one cannot know the exact time-frequency representation of a signal (i.e., what spectral components exist at what instants of time). What one can know is the time interval in which certain band of frequencies exists, which is a resolution problem. In DWT, this principle still holds but it is manipulated to achieve the optimal time and frequency resolutions at different frequency ranges.

This adjustment of the resolutions is inherent in wavelet transform as the wavelet basis is stretched or compressed during the transform. A high scale corresponds to a more "stretched" wavelet having a longer portion of the signal being compared with it. This would result in the slowly changing coarser feature of the signal to be determined accurately. On the contrary, a low scale uses compressed wavelet to sift out rapidly changing details that correspond to high frequencies. Compressed wavelet provides the necessary precision time resolution while compromising the frequency resolution. This ability to expand function or signal with different resolutions is termed as multiresolution analysis, which forms the cornerstone of many wavelet applications.

Armed with this capability, wavelet transform is used in many applications including signal suppression where certain parts are suppressed to highlight the remaining portion. The highlighted portion can either be low or high frequency. Another popular application is denoising where it is used to recovering signal from samples corrupted by noise. This is very effective when the noise energy is concentrated in different scales from those of the signal. In addition, the relative scarceness of wavelet representation allows unnecessary information to be discarded without compromising the original intent. This is heavily exploited in data compression 

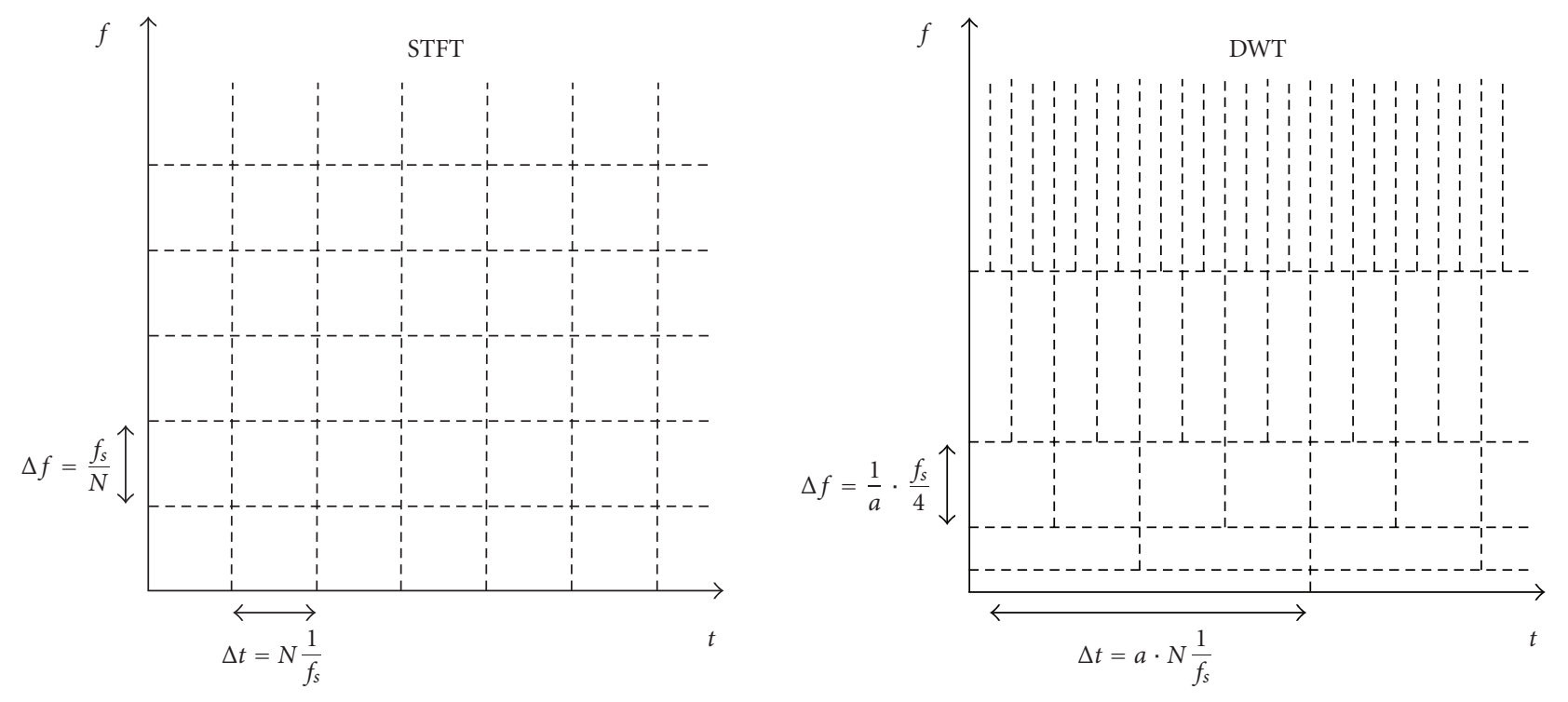

FIGURE 1: Comparison of time and frequency resolutions ( $f_{s}$ : sampling frequency; $N$ : number of sample points per analysis window).

especially in the storage and handling of images. Last but not least, the localization property of wavelet enables discontinuities or breakdown points to be easily and vividly identified. It is therefore widely applied for detection of the onset of certain events and to pin down the exact instant of the occurrence. Section 3 describes several power quality applications that make use of these capabilities.

\section{WAVELET APPLICATIONS IN POWER QUALITY}

The ability of wavelet transform in segregating a signal into multiple frequency bands with optimized resolutions makes it an attractive technique for analyzing power quality waveform. It is particularly attractive for studying disturbance or transient waveform, where it is necessary to examine different frequency components separately. This section discusses several popular uses of wavelet transform in the analysis of power quality disturbances.

\subsection{Characterization of voltage transients}

The time and space localization property of wavelet transform makes it highly suitable for analysis of discontinuities or abrupt changes in signal. In power systems, there are many voltage transients due to lightning strikes, equipment switching, load turning-on, and faults. With multiresolution analysis, the DWT provides a logarithmic coverage of the frequency spectrum as depicted in Figure 1. This has been shown to be useful in characterizing voltage transients caused by capacitor switching and faults [10]. Figure 2 shows how two voltage transient waveforms can be expanded into various levels (scales) corresponding to several frequency bands. In level 1, which is the highest frequency, several short bursts are observed for capacitor switching. Compared to the two distinct and separated bursts for fault, this can be used as the discriminating feature between the events. In addition, significant ringing is observed at level 4, which may be the system natural frequency that is significantly affected by the switched capacitor.

In the above example [10], there is no redundant information being used in the analysis as only one lowfrequency scale (highest scale) is used alongside the other high-frequency scales. This corresponds to one approximation term $A_{j 0}$ and multiple detail terms $D_{j}$ as defined in Figure 14, where $j_{0}$ is the total number of decomposition levels. However, some redundancies may be useful as they may give more obvious discriminating patterns. In [11], all the approximation terms $A_{j}$ in successive decomposition levels are also employed alongside the detail terms $D_{j}$ to form the discriminating patterns between fault transient and capacitor switching transient.

Similar wavelet expansion approach is also being proposed for analyzing current drawn by arc furnace [12]. The wavelet expansion helps to identify which frequency ranges the disturbance energy is concentrated. The same technique is also applied to inrush, fault, and load currents for differentiating between transformer magnetization inrushes, internal short circuit faults, internal incipient faults as well as external short circuit faults and load changes [13]. The reconstructed bands of signals from wavelet coefficients in the respective scales form the unique patterns necessary for discrimination.

\subsection{Characterization of short-duration voltage variations}

Short-duration voltage variations, namely, dips, swells, and interruptions are commonly encountered in power systems. Turning on large loads such as induction motors or faults are known to cause these voltage variations that badly affect 


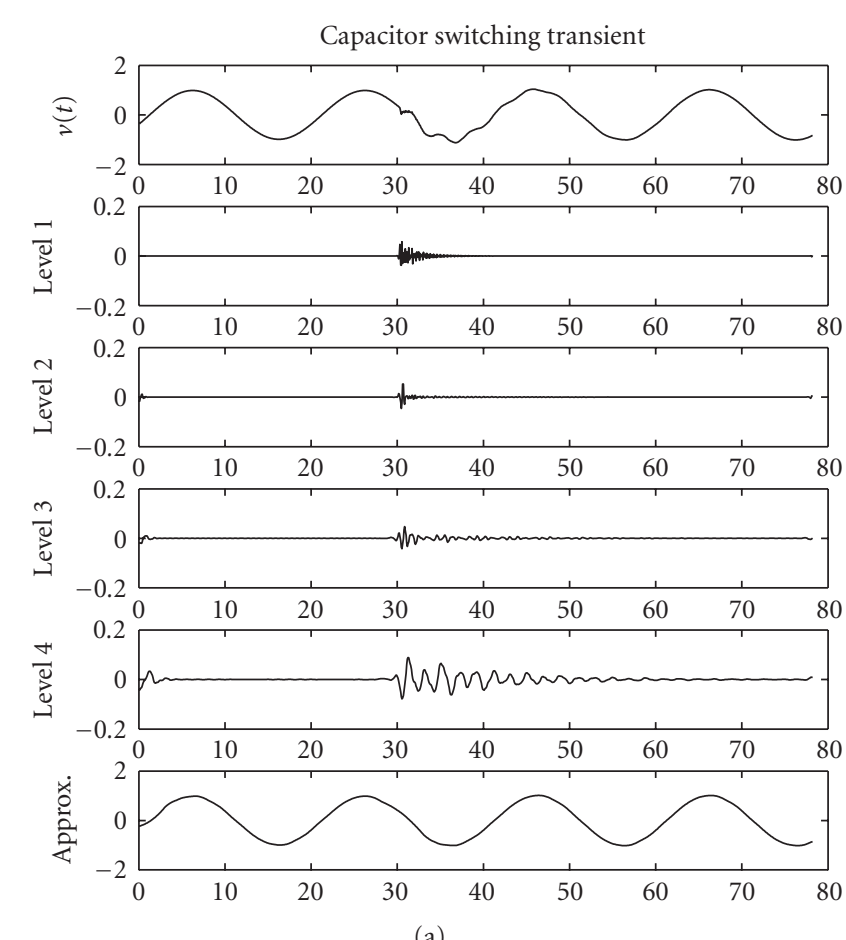

(a)
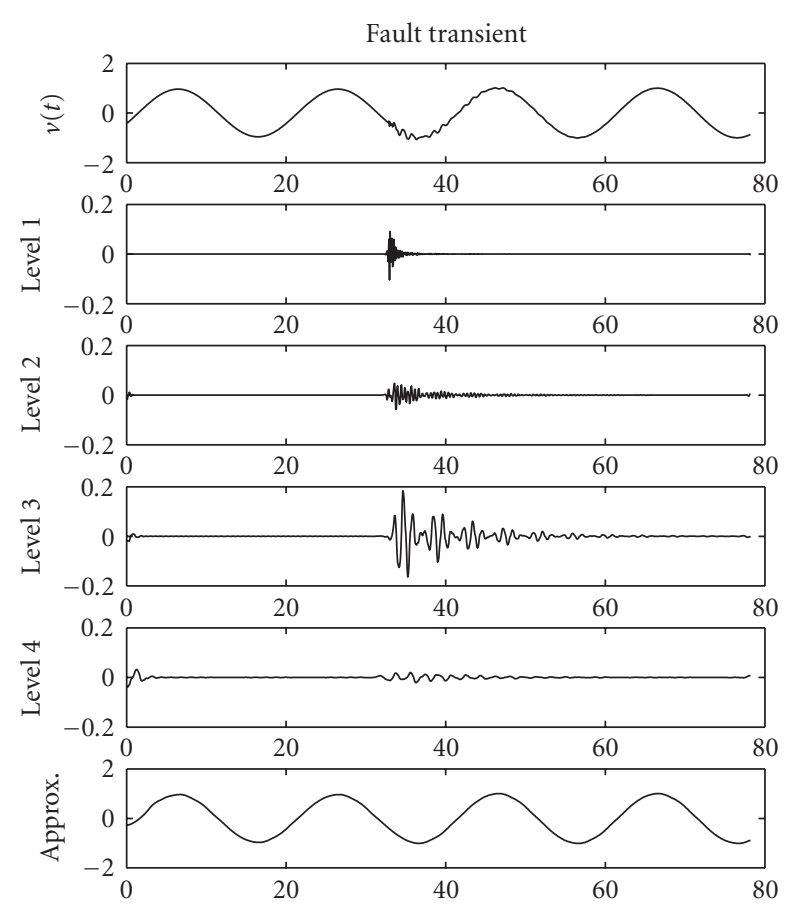

(b)

FIgURE 2: Wavelet expansion of voltage transients.

the operation of many modern electronic equipment. The important characteristics that indicate their severity are the magnitude and duration of the variations. Traditionally, RMS computation is used to derive the magnitude while the duration is taken as the time period the RMS magnitude stays below/above certain threshold $(<90 \%$ for dips and $>110 \%$ for swells. Although RMS method is generally considered as sufficient, the wavelet approach has been shown to produce more accurate results that would be useful for determining the causes of such variations.

Figure 3 shows the waveform of a short-duration voltage dip (70\%; 5 cycles) followed by a 10 -cycle interruption in (a), and the corresponding characterization using wavelet method. First, (b) and (c) shows the use of CWT in sifting out two frequency components of $50 \mathrm{~Hz}$ and $650 \mathrm{~Hz}$ and constructing their respective profiles [14]. The $650 \mathrm{~Hz}$ profile shows several sharp peaks denoting discontinuities, which are the occurring or ending instants of the disturbances. They are used to determine the durations of the dip and interruption. On the other hand, the $50 \mathrm{~Hz}$ profile shows magnitude of the dip and interruption, respectively. It can be shown that this approach works well too for very short voltage variation with duration less than half a cycle.

This method has also been suggested for analyzing highfrequency oscillatory transients. CWT is used to isolate the $1500 \mathrm{~Hz}$ component and if its profile shows sharp and short peaks, then the disturbance is one of the voltage variations. If it shows a long series of peaks, then it corresponds to highfrequency transients. The same argument can also be applied to the $650 \mathrm{~Hz}$ component for low-frequency transients.
Instead of CWT, it is more efficient to employ DWT without many compromises to the characterization accuracy. The multiresolution analysis capability of DWT ensures that fine time resolution is maintained at the high-frequency bands for determining the occurring and ending instants. Although the time resolution at the low-frequency band loses precision, it is not used to determine the times and hence it is still sufficient to approximate the magnitude variations. This is illustrated by (d) and (e). (d) is the DWT detail coefficients, which contain the high-frequency details with fine time resolution for pinpointing the time instants, while (e) is the DWT approximation coefficients reflecting the magnitude change.

\subsection{Classification of various power quality events}

The different levels of wavelet coefficient over the scales can be interpreted as uneven distribution of energy across the multiple frequency bands. This distribution forms patterns that have been found to be useful for classifying between different power quality events. If the selected wavelet and scaling functions form an orthonormal (independent and normalized) set of basis, then the Parseval theorem relates the energy of the signal to the values of the coefficients. This means that the norm or energy of the signal can be separated according to the following multiresolution expansion:

$$
\int|f(t)|^{2} d t=\sum_{k}\left|A_{j_{0}}(k)\right|^{2}+\sum_{j \leq j_{0}} \sum_{k}\left|D_{j}(k)\right|^{2} .
$$

These squared wavelet coefficients were shown to be useful features for identifying power quality events. In [15], the 


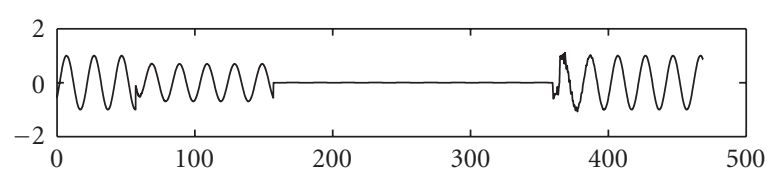

(a) Voltage waveform

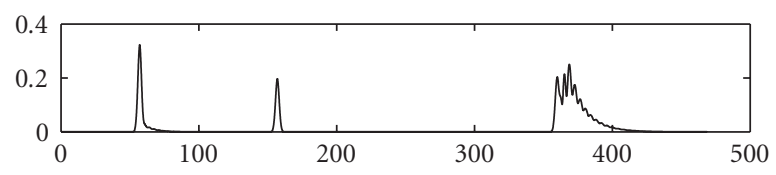

(b) CWT $650 \mathrm{~Hz}$ profile

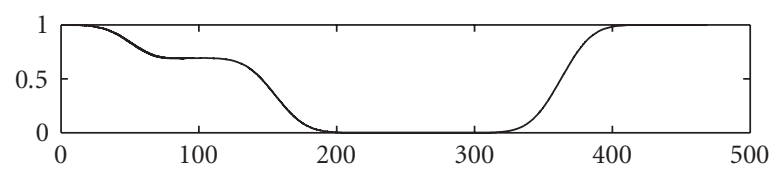

(c) CWT $50 \mathrm{~Hz}$ profile

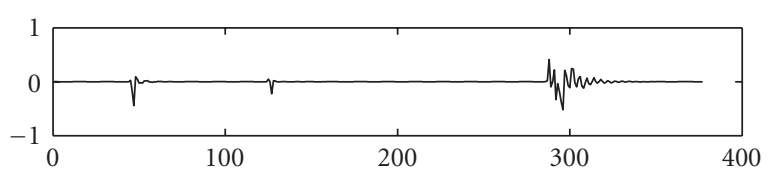

(d) DWT level 4 coefficients

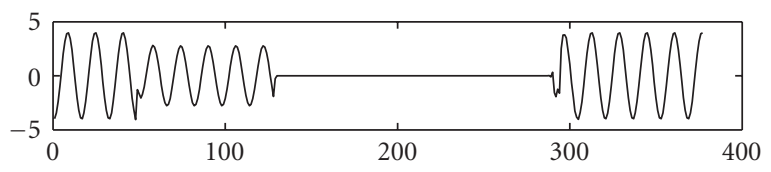

(e) DWT approx. coefficients

Figure 3: Characterization of a short-duration voltage dip.

statistics of these values are used to identify transformer energization, converter operation, capacitor energizing and restriking. The maximum value of the squared coefficients in each scale or its average is found to be different before, during, and after transformer energization. Changes in these values are used as the feature for its identification. Similarly, converter operation results in voltage notches, which are treated as discontinuities by wavelet transform and shown up in the high-frequency scales. Counting the number of highvalued squared coefficients over one fundamental period would lead to the event. Capacitor energization or breaker restriking on opening are known to cause rather dramatic voltage steps. When processed using DWT, high squared coefficients are found across various scales. Figure 4 shows a capacitor energizing transient waveform and the corresponding squared coefficients for three detail levels. The maximum values in each of the levels can be used as the feature to recognize the event. In [16], the averages or selections of coefficients are used as inputs to a self-organizing mapping neural network to distinguish between transients caused by load switching and capacitor switching.

Instead of using the maximum or average values, the energy distribution pattern in the wavelet domain can be computed as sums of the squared coefficients as in (6).
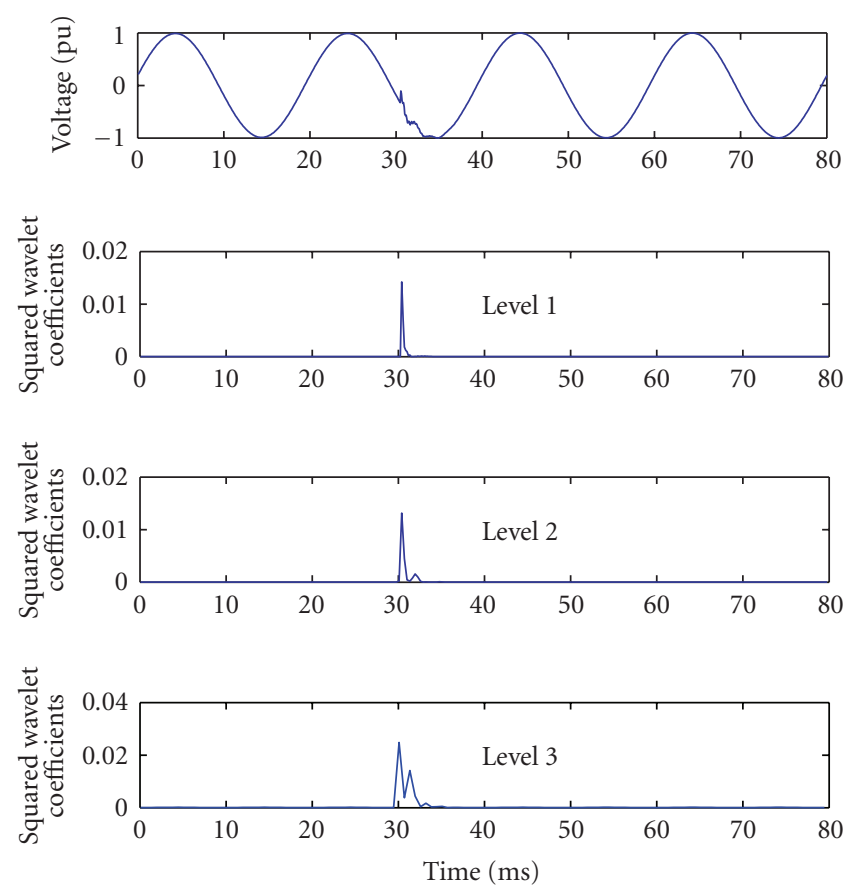

FIGURE 4: Capacitor-switching transient waveform and squared wavelet coefficients.

Figure 5 shows this energy distribution pattern for several commonly encountered power quality events. Differences between these patterns provide the differentiation features. Isolated capacitor switching shows more energy being distributed among the lower levels, corresponding to higher frequencies than the back-to-back switching. This reflects the differences between the high-frequency transients in the former condition and the low-frequency transients in the latter. Impulsive transient shows energy being generally confined to the highest frequency band (level 1). The pattern for voltage dip shows energy in the low-frequency region (level 5 ), which includes the fundamental frequency. However, the transients at the starting and ending instants manifest themselves as energy components in other lower scales (levels 3 and 4). These transients are not as pronounce when energizing transformer. Often, there are some uncertainties with the waveforms or patterns due to the varying system and component parameters. Hence, fuzzy reasoning is used to extend the identification rules derived from these energy distribution patterns [17]. Probabilistic neural network is another possible approach but it requires significant amount of data for training $[18,19]$.

\section{WAVELET METHOD DESIGN ISSUES}

The success of applying wavelet transform in various applications depends very much on several crucial design decisions. First, these decisions certainly have to be based on the objectives of the analysis. Although there can be many contrasting requirements, the bottom line can be narrowed to how accurate one can anticipate the nature of the analyzed signal. In 

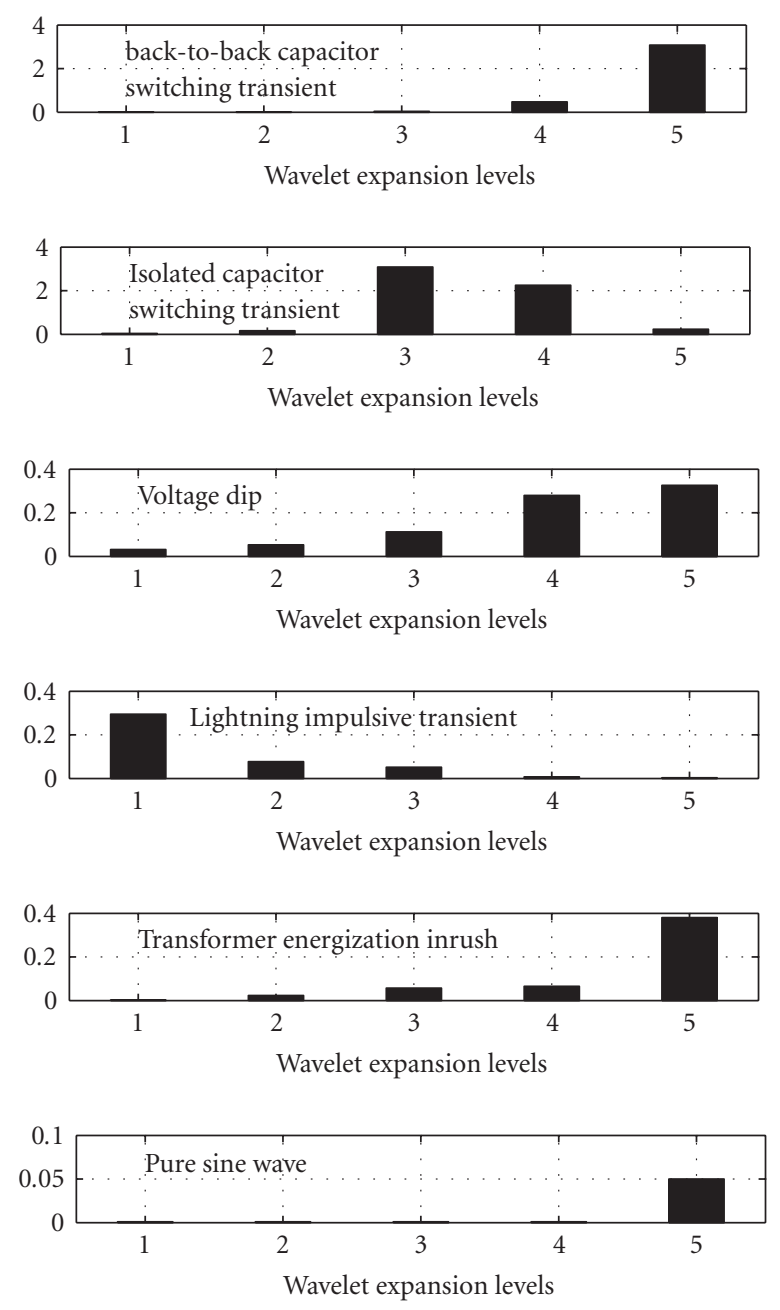

FIGURE 5: Energy distribution pattern in wavelet domain for various power quality events.

time-frequency decomposition, it is usually how exact one can anticipate the frequency contents of a signal that influences the choice of technique, the associated design settings, and the subsequent implementation. For wavelet transform, these are the choice of mother wavelet, CWT or DWT, and the number of expansion levels.

\subsection{Selection of mother wavelet}

Successful application of wavelet transform depends heavily on the mother wavelet. The most appropriate one to use is generally the one that resembles the form of the signal. This is particularly true for achieving good data compression ratio since a close resemblance would produce high coefficients in certain selective scales and near-zero coefficients in the remaining scales. However, this may not necessarily be as useful when forming patterns for identification and classification. Unique pattern for each event is more important than confining the coefficients to certain scales. Typically, if the representation can be spread across multiple scales, it tends to reduce the dependency on specific scales and thus helps to desensitize the identification and classification process. This would also make the process more robust and reduce erroneous identification.

There is a wide range of mother wavelets to choose from and each of them possesses unique properties as described in Appendix B. For power quality applications, it has been quoted to preferably be oscillatory, with a short support and has at least one vanishing moment $[11,19]$. The oscillatory feature is trivial as power networks are ac and many phenomena including transients are oscillatory in nature. A short support is a good trait as it keeps the number of high coefficients small. In addition to having less data to operate on, it also makes it easier to set thresholds for detection. Vanishing moments is another useful quality to have as it helps to suppress regular part of the signal, highlighting the sharp transitions. Unfortunately, support size and number of vanishing moments often go hand-in-hand and a compromise is necessary. Generally, most power quality applications would select a mother wavelet with short support but has one or two vanishing moments.

Among the several wavelet functions that were mentioned in the literature, the Daubechies family of wavelets are the most widely used $[12,13,15-18]$. This is perhaps due to its wavelets satisfying the necessary properties as described in the previous paragraph. Daubechies wavelets are also well known and widely used in other applications. It is flexible as its order can be controlled to suit specific requirements. Among the different $\mathrm{dbN}$ ( $N$-order) wavelets, $\mathrm{db} 4$ is the most widely adopted wavelet in power quality applications. It has sufficient number of vanishing moments to bring out the transients while maintaining a relatively short support to avoid having too many high-valued coefficients. Choosing the right mother wavelet often requires several rounds of trials, depending very much on the designer's experience and knowledge of the signal to be analyzed. Oftentimes, only subtle differences are observed from using one wavelet to another. The lack of explicit expressions for many wavelet functions also makes it difficult to compare them with mathematical rigours. Sometimes, it is the implementation issues such as the efficient DWT computation via FIR filtering that constitutes the overriding factor.

\section{2. $\quad C W T$ or DWT}

DWT can be viewed as a subset of CWT. This, on the outset, seems to favour CWT but as this is a redundant transformation, too much information may derail the identification and classification process. Hence, in the above illustrative examples, only one example uses CWT [14], while the others are all using DWT to take advantage of its provision of multiresolution analysis. In multiresolution analysis, the DWT process decomposes a signal into a discrete number of logarithmic frequency bands as shown on the left-hand side of Figure 6 [10]. At each level of decomposition or filtering and downsampling, the signal bandwidth is split into two halves of high and low frequencies. The low-frequency half is split further in subsequent decomposition or filtering. 


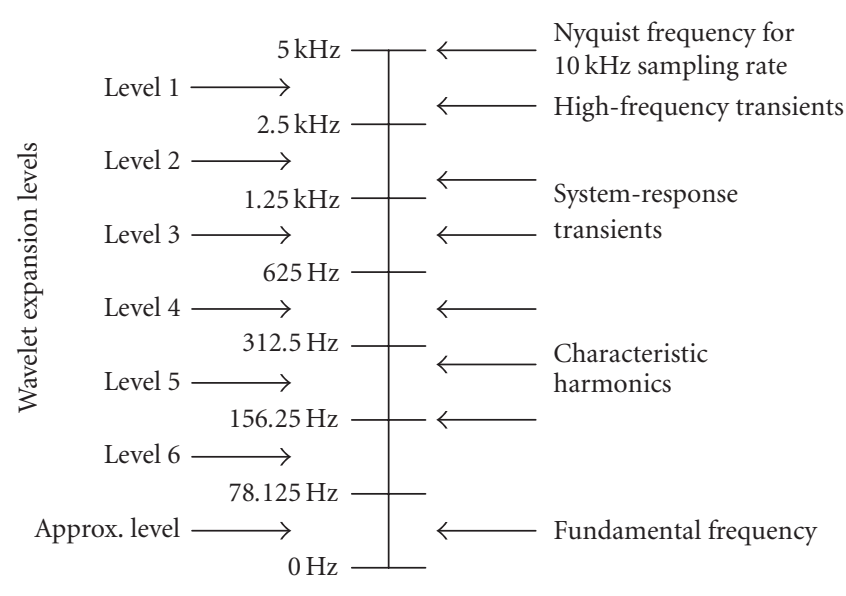

FIgURE 6: Frequency division of DWT filter for $10 \mathrm{kHz}$ sampling rate.

This rather rigid way of splitting the frequency bandwidths may pose some difficulties to certain applications.

On the right-hand side of Figure 6, the typical power quality phenomena of interest are listed [10]. Despite the rather rigid division in frequency, DWT is still deemed fit if the events of interest can be localized to within one or two bands. At high frequencies, the frequency bandwidths are wide leading to poor frequency resolutions. It can be seen that the high-frequency transients fall within a bandwidth between $2 \mathrm{kHz}$ and $3 \mathrm{kHz}$ and further processing is necessary to determine the predominant frequency if it is oscillatory. The wide bandwidth also admits many frequencies, making the filtering less selective at the high-frequency range. Therefore, if knowing specific frequency component is important, CWT or Fourier method is more suitable than DWT. However, if only an aggregate information within certain frequency bands are needed, DWT would be a more convenient and efficient choice.

\subsection{Number of decomposition levels}

The number of decomposition or expansion levels is very much related to the selection of CWT or DWT. For CWT, there is no rigid manner of decomposition, and hence the number of levels is arbitrary and as required. Frequently, it is decided according to the center frequency of the selected mother wavelet. For the CWT example shown in Figure 3, scales of 256 and 19.7 are selected for the $50 \mathrm{~Hz}$ and $650 \mathrm{~Hz}$ components, for using a complex mother wavelet cmor1-1.5 (center frequency of 1) and sampling rate of $12.8 \mathrm{kHz}(256$ samples per fundamental cycle). On the other hand, with limited levels in DWT, it has to be decided carefully and it depends on how many divisions are to be made to the lowfrequency ranges. Four to five levels of decomposition seem to be the most popular [13, 15-17], while some use seven to eight levels $[10,12]$, or even as many as thirteen levels [18]. In $N$ DWT decomposition levels, there will be $N-1$ detail levels and 1 approximation level. Most applications use both the detail and approximation levels but some use only the de- tail levels. The approximation level is almost always used to trace the fundamental frequency component only.

\subsection{Wavelet or Fourier}

It is inevitable that the wavelet techniques would be compared to the popular Fourier techniques. The Fourier theory is deeply entrenched in many areas of power system engineering, and this leads to a "risk" or "trap" that wavelet techniques are used to represent or mimic Fourier-based expressions. Fourier techniques rely on relatively good knowledge of the signal spectrum. The design of measurement and processing systems are heavily dependent on this knowledge. Otherwise, spectral leakage can be significant leading to the need for windowing, which adds to the implementation complexity.

In discrete Fourier transform (DFT), the window length has a pronounce effect as it determines the frequency resolution. The evaluated coefficients are basically magnitude and phase angle of each discrete frequency component. Wavelet techniques on the other hand are form of time-frequency analysis with predefined or accompanied windowing. Its coefficients denote information contained within successive bands of frequency. It is more forgiving for any slip-up in anticipating the frequency content of the signal. Therefore, it can be generalized that wavelet method is attractive when one is not absolutely certain about the frequencies that make up the signal. This is often the situation for voltage transient and wavelet methods are strongly advocated for analyzing transient signals with abrupt changes.

\subsection{Wavelet for harmonic and interharmonic analysis}

The ability to segregate between frequencies also leads to proposals to use wavelet transform in the analysis of harmonics and interharmonics. However, as these phenomena by definitions are sinusoids, it is always questionable if it is sensible to represent them using other basis functions besides the customary sine and cosine functions. Wavelet transform with some time information does possess the ability to track variations. However, it is arguable that this tracking can also be achieved through windowing such as in STFT.

To analyze harmonic and interharmonic distortion problems, it is necessary to know individual or groups of harmonics and interharmonics. In IEC Standard 61000-4-7 [20], a window length of 10 (or 12) cycles is recommended for use in 50 (or 60 ) $\mathrm{Hz}$ power systems, producing frequency-domain representations in $5 \mathrm{~Hz}$ bins. These $5 \mathrm{~Hz}$ bins are then combined to produce harmonic and interharmonic groupings and components for which compatibility levels and limits are specified.

As $5 \mathrm{~Hz}$ resolution is required at both high- and lowfrequency ranges, DWT is not suitable. An adapted version, called wavelet packet transform (WPT), can be used as the high-frequency details coefficients are also decomposed further at each subsequent level. This effectively creates a series of bandpass filters with relative similar bandwidths across the entire frequency plane. With proper selection of 
mother wavelet and number of decomposition levels, this approach has been shown to produce comparable results as those using DFT [21]. However, the design and implementation can be rather complex and it has yet to be proven to bring about much advantage when compared to DFT. In addition, harmonics and interharmonics are characteristically defined as sinusoids, making DFT the more convenient method, especially when results are to be checked against standard or guideline. Specifically, wavelet transform can be employed to track their variation, but as these phenomena are normally considered as steady-state or quasisteady-state, the usual DFT is an equally effective analysis method.

\section{WAVELET TRANSFORM AND RANK CORRELATION FOR IDENTIFICATION OF CAPACITOR-SWITCHING TRANSIENTS}

Among the many voltage disturbances in power systems, oscillatory transients caused by capacitor switching are commonly encountered as capacitors are used to improve the customers' load power factor or for utility voltage support. These transients typically take the form of underdamped response as follows:

$$
\begin{aligned}
V(t)= & A_{0} \cdot \sin \left(2 \pi f_{0} t+\varphi_{0}\right)+e^{-\alpha_{1} t} \cdot A_{1} \cdot \sin \left(2 \pi f_{1} t+\varphi_{1}\right) \\
& +e^{-\alpha_{2} t} \cdot A_{2} \cdot \sin \left(2 \pi f_{2} t+\varphi_{2}\right)+\cdots
\end{aligned}
$$

where the subscript 0 denotes the fundamental frequency, and the remaining subscripts refer to the oscillatory transients. Each transient component is characterized by its amplitude $A_{x}$, oscillating frequency $f_{x}$, and damping factor $\alpha_{x}$.

These characteristics are often used to identify and detect capacitor switching. The oscillating frequency and magnitude variation were used to determine the size and location of the shunt capacitor [22]. In [15], Santoso used the typical frequency and the variation of step voltage after switching to characterize capacitor switching transient. Despite these past efforts, differentiating capacitor switching transients from other disturbances remains a challenge. This is because the transient behaviour depends considerably on the system conditions and the capacitor. Particularly, variations in system conditions and capacitor power ratings alter these characteristics, posing challenges to measurement and detection techniques that focus on these quantities. Wavelet techniques, with their bandpass property, are therefore more robust than Fourier methods as they are less frequency selective. The transient amplitude and the manner it decays away are heavily affected by the system and component variations. This impact can be largely nullified by using other measures such as the ranks instead of the absolute magnitudes of the captured transient waveform. This section introduces the use of rank correlation for analyzing the underdamped response of the transient component as a mean to identify capacitorswitching events.

\subsection{Extracting the transient component}

Energizing a capacitor bank typically results in two major transient components, inrush transient and energizing transient. The former is due to an initial downward surge of the voltage as the charged system capacitance tries to transfer its charges to the uncharged capacitor. This transient can be significant when turning on large capacitors and it also occurs when turning on loads that are fitted with power factor correction capacitor. This inrush transient is typically of high frequencies in tens of $\mathrm{kHz}$, making it difficult to measure. Hence, it is not commonly used for identification. In addition, capacitors are often fitted with $1 \mathrm{mH}$ inductance to limit this inrush, affecting the measurement.

After the initial inrush, the system would eventually charges up the combined capacitance. This charging causes another voltage and current surge, cumulating to the energizing transient. It is oscillatory but damped out gradually by the system resistance. Unlike inrush transient, it is more substantially affected by the system conditions. Its frequency is much lower, at around $1 \mathrm{kHz}$, and it can be readily measured and used for identification. However, finding the exact frequency is difficult unless all of the system parameters are known. Even if relying on prior knowledge of the system or past measurements, it is more practical to estimate the probable frequency range. This then requires an analysis method that is not heavily dependent on having precise information on this frequency. Wavelet methods fit this requirement as they are band-limited filters and not confined to any specific frequency.

In this method, CWT is preferred over DWT due to its more flexible frequency selection. DWT, with its dyadic calculation structure, confines its scale and frequency band to discrete values, making it difficult to contain all transient information within a single band for identification. The centerfrequency of a CWT scale is adjusted to match as closely as possible to the expected dominant frequency of the energizing transient. In addition, with its inherent redundancy, the time resolution is maintained ensuring sufficient data is available in all bands for use in the identification.

Once the dominant frequency is trapped, its magnitude variation $e^{-\alpha_{1} t} \cdot A_{1}$ is reflected by the change in the energy content of the particular frequency band. Such information on the voltage $V_{c}(t)$ at a particular scale $s_{c}$ and time instant $t_{0}$ can be obtained using the following expressions:

$$
\begin{aligned}
\mathcal{W}\left\{V_{c}\left(t_{0}, s_{c}\right)\right\} & =\int_{-\infty}^{+\infty} V_{c}(t) \frac{1}{\sqrt{s_{c}}} \psi^{*}\left(\frac{t-t_{0}}{s_{c}}\right) d t \\
& =\left\langle V_{c}, \psi_{t_{0}, s_{c}}\right\rangle=V_{c} * \bar{\psi}_{s_{c}}\left(t_{0}\right) .
\end{aligned}
$$

The corresponding energy density at this scale and at this time instant can be calculated as

$$
P_{W}\left(t_{0}, s_{c}\right)=\left|\mathcal{W}\left\{V_{c}\left(t_{0}, s_{c}\right)\right\}\right|^{2}
$$

With this energy density definition, the energy from half a cycle of the voltage waveform, which indirectly reflects the 
magnitude of this band, is

$E_{W}\left(s_{c}, t_{0}\right)=\int_{t_{0}}^{t_{0}+T_{1} / 2} P_{W}\left(t, s_{c}\right) d t=\int_{t_{0}}^{t_{0}+T_{1} / 2}\left|\mathcal{W}\left\{V_{c}\left(t, s_{c}\right)\right\}\right|^{2} d t$,

where $T_{1}$ is the period of the oscillatory transient. By sliding the computation window over time, changes in the energy content reflects the magnitude variation. The magnitude of this energy varies with its initial value $A_{1}$, which depends among many parameters on the point-on-wave when the capacitor is switched.

\subsection{Rank correlation}

Rank correlation is a kind of nonparametric statistical method that evaluates the similarity between two signals through their ranks. It is used here to evaluate the similarity between the variation in the transient amplitude and that of a predefined signature waveform. The correlation gives a value close to 1 if they match, verifying that the disturbance is similar to the signature. Instead of comparing the absolute magnitudes, rank correlation evaluates whether the shape of a signal fits that of another signal. This method is immune to the measurement methods as it only concerns with the shape and not on the actual value. It is easy to implement and appear to be a good choice for comparing the amplitude variation of capacitor-switching transients. There are two main types of rank correlation methods, the Spearman and the Kendall [23], and the former is used in this method.

Spearman rank correlation is a distribution-free analogy of correlation analysis. It compares two independent random variables, each at several levels (which may be discrete or continuous). It judges whether the two variables covary (i.e., vary in similar direction) or as one variable increases, the other variable tends to increase or decrease. Spearman rank correlation works on ranked (relative) data. The smallest value is replaced with a 1 , the next smallest with a 2 , and so on. It measures the nonlinear relationship or the similarity between two variables despite their different magnitudes. It is suitable for use with skewed data or data with extremely large or small values. Ties are assigned if some variables have identical values, and the average of their adjacent ranks is used in the comparison. With "ties," the Spearman rank correlation coefficient is calculated as

$$
\begin{aligned}
\rho_{s}= & {\left[1-\frac{6}{N^{3}-N}\left[\sum_{i=1}^{N}\left(R_{i}-S_{i}\right)^{2}+\frac{1}{12} \sum_{k}\left(f_{k}^{3}-f_{k}\right)\right.\right.} \\
& \left.\left.+\frac{1}{12} \sum_{m}\left(g_{m}^{3}-g_{m}\right)\right]\right] \\
& /\left[\left[1-\frac{\sum_{k}\left(f_{k}^{3}-f_{k}\right)}{N^{3}-N}\right]^{1 / 2}\left[1-\frac{\sum_{m}\left(g_{m}^{3}-g_{m}\right)}{N^{3}-N}\right]^{1 / 2}\right],
\end{aligned}
$$

where $N$ is the length of the two variables; $R_{i}$ and $S_{i}$ are the ranks of respective variables; $f_{k}$ and $g_{m}$ are the number of ties in the $k$ th or $m$ th group of ties among the $R_{i}$ 's or $S_{i}$ 's. The co-

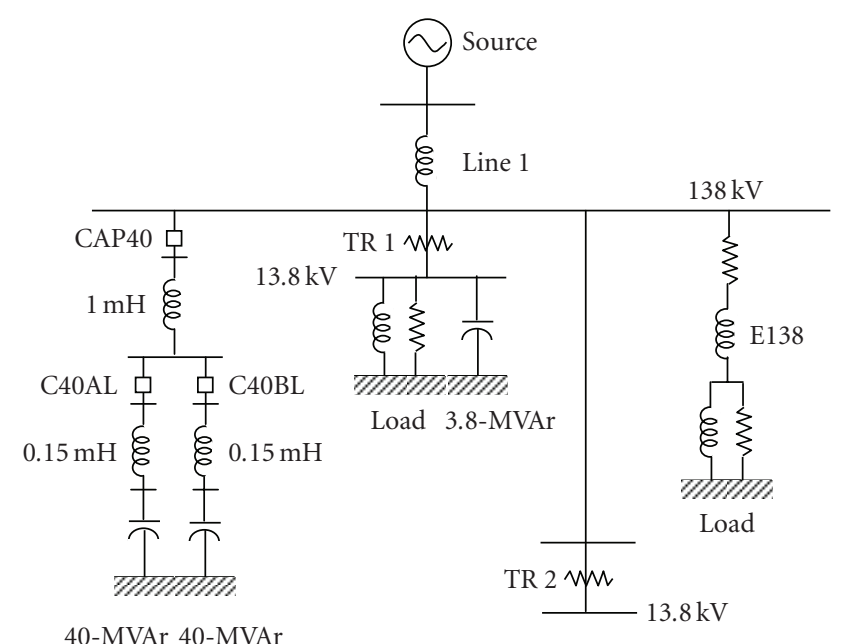

FIGURE 7: Single-line diagram of a $138 \mathrm{kV} 60 \mathrm{~Hz}$ illustrative system.

efficient $\rho_{s}$ indicates agreement. A value near 1 indicates good agreement while a value near zero or negative, poor agreement.

\subsection{Dynamic simulations and verifications}

A $138 \mathrm{kV} 60 \mathrm{~Hz}$ test system as shown in Figure 7 is used to illustrate this method [24]. Two 40 MVAr capacitor banks at the $138 \mathrm{kV}$ busbars are used to simulate the two types of capacitor switching-isolated and back-to-back. The threephase fault current at the $138 \mathrm{kV}$ bus is approximately $13.7 \mathrm{kA}$, giving a short circuit capacity of about 1890.6 MVA. For the study of switching transient, the test system can be simplified to a Thevenin equivalent with a series resistance $R_{s}$ of $0.58 \Omega$, series inductance $L_{s}$ of $15.39 \mathrm{mH}$ giving $X_{s}$ of $5.8 \Omega$ and $X / R$ ratio of 10 . The system stray capacitance is taken to be $1200 \mathrm{nF}$ and used only in the analysis of isolated switching. For the back-to-back switching, one of the two $40 \mathrm{MVAr}$ capacitor banks is assumed to be already connected when the other one is switched.

Dynamic simulations are carried out using Matlab/ Simulink. The transient waveforms are processed using CWT followed by rank correlation to identify if they are caused by capacitor switching. $\mathrm{db} 2$ is the mother wavelet due to its simplicity. With a center-frequency of 0.6667 and sampling frequency of $15.36 \mathrm{kHz}$, a wavelet scale of 22.8 is selected, giving a pseudofrequency of $449.12 \mathrm{~Hz}$. This is close to the estimated response frequency of the signature at $450 \mathrm{~Hz}$. Figure 8 shows the oscillatory transients from the energization of a 40 MVAr capacitor. For the signature, it is assumed that the capacitor is switched at the voltage peak producing the biggest transient. The corresponding amplitude variation of the signature and the transients derived using (10) are shown in Figure 9. Due to the differences in their amplitudes, absolute correlation of the phases with the signature would not produce good agreement. Particularly, phase A with small transient showing the biggest difference from the signature would produce a rather low coefficient. In 


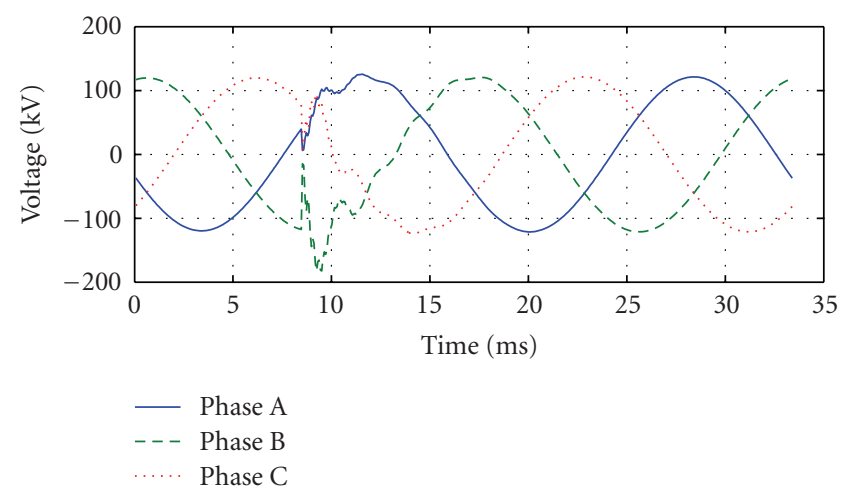

FIGURE 8: Voltage waveforms of a capacitor-switching transient.

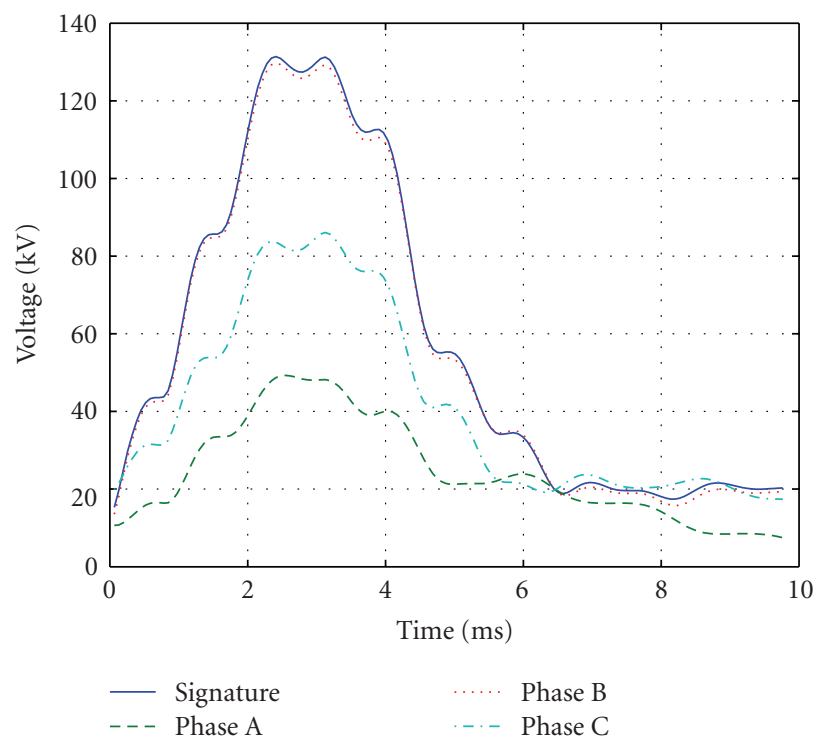

Figure 9: Amplitude variations of signature and transient.

a three-phase system, the instantaneous magnitudes of the phase voltages are generally different, with one phase low, close to zero while the other two phases much higher. Therefore, the transient on that particular phase would be much smaller compared to the other two.

On the other hand, if the ranks of the signature and transients are compared as shown in Figure 10, the correlation coefficients are much higher and more consistent. As can be seen from the graphs, the ranks for the phases very much coincide with that of the signature despite the amplitude differences. Even for phase A with small transient amplitude, the correlation remains good and much better than the absolute correlation of Figure 9. However, to maintain high accuracy, only the two most significant phases are used for identification. Their average value is compared to some threshold, such as 0.9 , to deduce on whether the transient is caused by capacitor switching. In the following subsections, several simulations are carried out to verify the robustness of this wavelet-based rank correlation method.

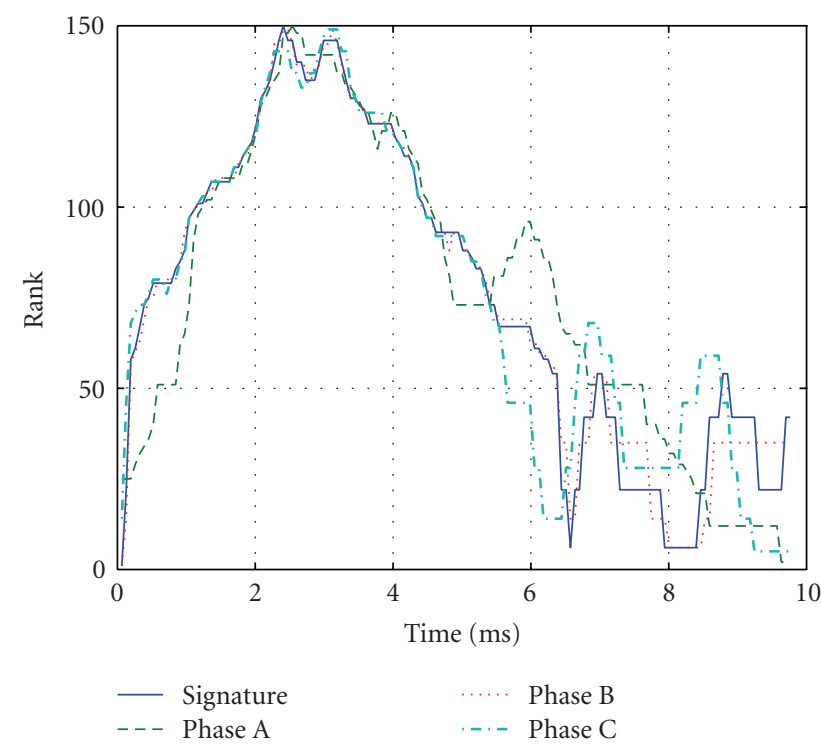

FIgURE 10: Ranks of signature and transient.

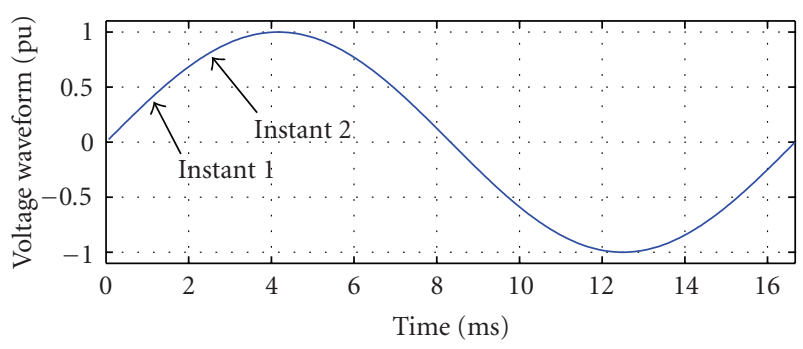

FIGURE 11: Switching instants on phase A voltage waveform.

\subsubsection{Effect of different switching instants}

Figure 11 shows two point-on-wave instants of one of the phases when the capacitor is energized. Two sets of simulation results are obtained as shown in Tables 1, 2, and 3 for isolated and back-to-back capacitor switching. For each of the cases, it is obvious that one phase shows a poorer correlation compared to the other two. The averages shown in the rightmost column are derived from the two phases with the highest coefficients. A result close to 1 denotes close resemblance to the signature, suggesting that the disturbance is a capacitor-switching transient. Different switching instants mean that different instantaneous voltage being impressed on the uncharged capacitor when it is switched. In addition, the phases are phase-shifted from each other, causing the rank correlation results to vary between the phases and from one instant to another. Nonetheless, two of the phases always show significant transients that allow the rank correlation method to produce noticeable result, even though the instants are different from that of the signature at voltage peak. 
TABLE 1: Results of isolated capacitor switching.

\begin{tabular}{l|ccccc}
\hline Capacitor size & Switching instant & Phase A & Phase B & Phase C & Average of two highest phases \\
\hline \multirow{2}{*}{$20 \mathrm{MVAr}$} & 1 & 0.786 & 0.933 & 0.691 & 0.860 \\
& 2 & 0.956 & 0.797 & -0.362 & 0.876 \\
\hline \multirow{2}{*}{$40 \mathrm{MVAr}$} & 1 & 0.887 & 0.989 & 0.929 & 0.959 \\
& 2 & 0.971 & 0.967 & 0.364 & 0.969 \\
\hline \multirow{2}{*}{$60 \mathrm{MVAr}$} & 1 & 0.897 & 0.951 & 0.932 & 0.941 \\
& 2 & 0.956 & 0.940 & 0.541 & 0.948 \\
\hline \multirow{2}{*}{$80 \mathrm{MVAr}$} & 1 & 0.825 & 0.916 & 0.886 & 0.901 \\
& 2 & 0.914 & 0.907 & 0.493 & 0.911 \\
\hline
\end{tabular}

TABLE 2: Results of back-to-back capacitor switching with 40 MVAr connected.

\begin{tabular}{l|ccccc}
\hline Capacitor size & Switching instant & Phase A & Phase B & Phase C & Average of two highest phases \\
\hline \multirow{2}{*}{$20 \mathrm{MVAr}$} & 1 & 0.558 & 0.952 & 0.747 & 0.850 \\
& 2 & 0.955 & 0.833 & -0.414 & 0.894 \\
\hline \multirow{2}{*}{$40 \mathrm{MVAr}$} & 1 & 0.712 & 0.922 & 0.864 & 0.893 \\
& 2 & 0.918 & 0.890 & -0.006 & 0.904 \\
\hline \multirow{2}{*}{$60 \mathrm{MVAr}$} & 1 & 0.689 & 0.847 & 0.813 & 0.830 \\
& 2 & 0.833 & 0.842 & 0.530 & 0.838 \\
\hline \multirow{2}{*}{$80 \mathrm{MVAr}$} & 1 & 0.649 & 0.798 & 0.781 & 0.789 \\
& 2 & 0.771 & 0.793 & 0.066 & 0.782 \\
\hline
\end{tabular}

\subsubsection{Effect of different capacitor ratings}

Tables 1, 2, and 3 also show the results of switching capacitors of lower and higher ratings than the 40 MVAr used to generate the signature. Nevertheless, the correlation results remain high above 0.75 , and they are still reasonably good for identifying capacitor switching. This is because although different capacitor size would result in different characteristic frequency, the relative frequency variation is small. In these cases, the frequency variations are smaller than $\pm 50 \mathrm{~Hz}$ in isolated switching cases and $\pm 100 \mathrm{~Hz}$ in back-to-back cases. These ranges are still very much covered by the selected CWT bandwidth, producing satisfactory rank correlation results. The declining trend indicates that the correlation deteriorates as the actual system response moves away from that of the signature. A lower threshold value may be necessary if there exists such large uncertainties.

\subsubsection{Isolated or back-to-back switching}

Comparing Tables 2 and 3 to Table 1, the influence of existing capacitance in the network can be observed. In Table 2, a 40 MVAr capacitor is assumed to be in service when another capacitor is switched. In Table 3, the connected capacitor is assumed to be twice as big at 80 MVAr. Generally, different responses are expected but the characteristics of these switching transients tend to overlap in both frequency and time. Therefore, the selected signature, which is derived from isolated switching of a 40 MVAr capacitor, is still largely effective for back-to-back switching except for a few conditions where the size of the switched capacitor also differs from the 40 MVAr used in the signature.

\subsubsection{Effects from different system conditions}

It is always difficult to be completely certain about all of the system parameters. Any uncertainty may affect the identification accuracy. Table 4 shows the correlation results when the system inductance is half or double of that used to derive the signature. The results confirm the robustness of the method as the rank correlation coefficients remain good, greater than 0.9. Although these variations change the transient damping time constant from 6 milliseconds to 9 milliseconds, it however does not affect the performance of the method. Similar studies were also conducted for different system resistances and system capacitances, and the correlation remains good. For the cases on system capacitance, the uncertainties show weaker impact compared to those from back-to-back switching. In such cases, the connected capacitor would dominate the system capacitance and affects the transient characteristics. However, as shown in Table 2, the proposed method is still applicable albeit needing a lower threshold.

\subsubsection{Noncapacitor-switching transients}

To further verify the validity of the method, transients caused by other switching events are also simulated and used to test its rejection capability. The reactor switching is the energization of a 40 MVAr reactor at the $138 \mathrm{kV}$ busbar. The transformer switched is rated at $20 \mathrm{MVA} 138 / 3.8 \mathrm{kV}$, while the 
TABLE 3: Results of back-to-back capacitor switching with 80 MVAr connected.

\begin{tabular}{l|ccccc}
\hline Capacitor size & Switching instant & Phase A & Phase B & Phase C & Average of two highest phases \\
\hline \multirow{2}{*}{$20 \mathrm{MVAr}$} & 1 & 0.174 & 0.781 & 0.136 & 0.477 \\
& 2 & 0.765 & 0.672 & -0.486 & 0.718 \\
\hline \multirow{2}{*}{$40 \mathrm{MVAr}$} & 1 & 0.315 & 0.788 & 0.751 & 0.769 \\
& 2 & 0.744 & 0.812 & -0.336 & 0.778 \\
\hline \multirow{2}{*}{$60 \mathrm{MVAr}$} & 1 & 0.477 & 0.826 & 0.761 & 0.794 \\
& 2 & 0.800 & 0.817 & -0.298 & 0.809 \\
\hline \multirow{2}{*}{$80 \mathrm{MVAr}$} & 1 & 0.575 & 0.841 & 0.770 & 0.805 \\
& 2 & 0.829 & 0.826 & -0.258 & 0.827 \\
\hline
\end{tabular}

TABLE 4: Results with changes to system parameters.

\begin{tabular}{l|ccccc}
\hline System change & Switching instant & Phase A & Phase B & Phase C & Average of two highest phases \\
\hline \multirow{2}{*}{$50 \% L_{s}$} & 1 & 0.847 & 0.959 & 0.955 & 0.957 \\
& 2 & 0.941 & 0.963 & -0.008 & 0.952 \\
\hline \multirow{2}{*}{$00 \% L_{s}$} & 1 & 0.811 & 0.867 & 0.903 & 0.885 \\
& 2 & 0.849 & 0.904 & 0.213 & 0.876 \\
\hline \multirow{2}{*}{$50 \% R_{s}$} & 1 & 0.886 & 0.988 & 0.935 & 0.962 \\
& 2 & 0.970 & 0.970 & 0.369 & 0.970 \\
\hline \multirow{2}{*}{$50 \% R_{s}$} & 1 & 0.892 & 0.989 & 0.916 & 0.953 \\
& 2 & 0.971 & 0.965 & 0.369 & 0.968 \\
\hline \multirow{2}{*}{$200 \% C_{s}$} & 1 & 0.884 & 0.969 & 0.907 & 0.939 \\
& 2 & 0.971 & 0.944 & 0.399 & 0.957 \\
\hline
\end{tabular}

TABLE 5: Results of other disturbances.

\begin{tabular}{l|crrcc}
\hline Transient events & Cases & Phase A & Phase B & Phase C & Average of two highest phases \\
\hline \multirow{2}{*}{ Reactor switching } & 1 & -0.088 & 0.830 & -0.645 & 0.371 \\
& 2 & 0.882 & -0.241 & -0.595 & 0.321 \\
\hline \multirow{2}{*}{ Transformer energization } & 1 & -0.036 & 0.849 & -0.667 & 0.407 \\
& 2 & 0.849 & -0.282 & -0.550 & 0.284 \\
\hline \multirow{2}{*}{ Line energization } & 1 & -0.060 & 0.815 & -0.604 & 0.377 \\
& 2 & 0.906 & -0.097 & -0.587 & 0.404 \\
\hline
\end{tabular}

line energized is $50 \mathrm{~km}$ long, both connected to the $138 \mathrm{kV}$ busbar. The results in Table 5 show low average correlations that can be easily rejected by setting an appropriate threshold. Generally, the characteristic frequencies of these transients are not close to that of the capacitor switching. The rank correlation results would therefore be low or even negative. For some phases and at certain switching instants, the correlation appears high at about 0.85 . However, closer inspection reveals that the transients are very small, and can be discounted in the first place. The good correlation there is due to the very low energy content having similar profile as that of signature. Fortunately, the other two phases show poor correlation, helping to reject their identification. From these results, it is illustrated that by selecting an appropri- ate threshold such as 0.70 , the majority of these disturbances can be differentiated from the transients caused by capacitor switching.

\section{CONCLUSIONS}

Wavelet transform is quickly becoming the choice method for processing power quality data. This is due to its excellent time and frequency localization capability, and the flexibility in implementation. However, using wavelet transform can be involving, especially at the design stage. The many flexible features translate into more decisions that need to be made. Selection of mother wavelet, using CWT or DWT and the number of decomposition levels are the three 
most important decisions that can affect its performance significantly. Wavelet transform is more forgiving than other methods when the form of the signal is not clearly known. It is ideal for handling phenomena with fast changing or abrupt transients. In this paper, it is shown to be effective for characterizing and classifying switching transients, fault transients, and short-duration voltage variations. The response frequency of these disturbances tends to fluctuate with changes in the system but generally remains within certain band that can be easily covered by wavelet techniques. These characteristics are exploited in a new capacitor switching transient identification method. The method adopts the use of rank correlation in place of the customary absolute correlation, overcoming several challenges that are brought about by uncertainties in the system conditions. However, Wavelet method is found to be not useful for phenomena whose characteristics are customarily defined using Fourier or other basis functions, such as harmonics and interharmonics. As the understanding of wavelet transform grows, there will be more attempts at applying it to power quality analysis but it remains to be seen if it can be as successful on other issues as on transients.

\section{APPENDICES}

\section{A. WAVELET TRANSFORMS}

Wavelet transform is carried out by convolving a wavelet with a signal or function. The wavelet can be manipulated in two ways: stretched or squeezed (scaled) and moved to various locations on the signal. If it matches the signal well at a specific scale and location, then a large transform value is obtained. However, if they do not correlate well, a low value ensues. The transform is usually computed at various locations on the signal and at various scales. It is done in a smooth continuous fashion for the continuous wavelet transform (CWT) or in discrete steps for the discrete wavelet transform (DWT).

Compared to other time-frequency analysis, CWT decomposes signal with less restriction on its resolution. It can operate at any scale, up to the highest scale that is limited only by the Nyquist sampling theorem [9]. CWT is also continuous in terms of time shifting. During computation, the scaled mother wavelet is shifted smoothly over the full domain of the signal. Accordingly, a one-dimensional signal is translated into a two-dimensional time-frequency representation by the coefficients of CWT. This smooth transition of the scaled mother wavelet implies that there are many overlaps in the transform making the CWT representation highly redundant.

Instead of the highly redundant CWT, DWT is often used as it is more efficient computationally and requires less memory storage. Unlike CWT where the expansion is performed on any arbitrary scale, DWT follows a certain discrete expansion pattern determined by the selection of a factor $a_{0}$. The most widely used pattern is called dyadic expansion with $a_{0}=2$ and the expansion is implemented for scales $a=a_{0}^{j}$, where $j=1,2,3, \ldots$. The information in the high- frequency bands as carried by the details $D_{j}$ is computed as

$$
D_{j}[n]=\mathcal{W}\left\{f\left[a_{0}^{j}, n\right]\right\}=\sum_{m=0}^{N-1} f[m] \cdot \frac{1}{\sqrt{a_{0}^{j}}} \psi^{*}\left(\frac{m-n}{a_{0}^{j}}\right),
$$

where $N$ is the total number of samples of the analyzed signal. Similarly, the information in the lowfrequency band as carried by the approximations $A_{j}$ are coefficients of DWT with the scaling function

$$
A_{j}[n]=\mathcal{L}\left\{f\left[a_{0}^{j}, n\right]\right\}=\sum_{m=0}^{N-1} f[m] \cdot \frac{1}{\sqrt{a_{0}^{j}}} \phi^{*}\left(\frac{m-n}{a_{0}^{j}}\right) .
$$

\section{A.1. Frequency responses of wavelet and scaling functions}

The stretching or compressing of wavelet and scaling functions to derive high- and low-frequency information is akin to filtering through a highpass filter and a lowpass filter. Indeed, wavelet and scaling functions can be interpreted as impulse responses of highpass and lowpass filters, respectively. Figure 12 shows a widely used mother wavelet, $\mathrm{db} 2$ and its Fourier transforms for several normalized scales. They resemble the frequency responses of bandpass filters. The bandwidth and center frequency of each pass band vary with the scale $a$. At low scale, corresponding to high frequency, the pass band is wide with high center frequency. As $a$ increases and approaches 1 , the center frequency reduces and the bandwidth becomes narrower too, as the frequency response shifts to the left. This demonstrates that the frequency resolution or localization becomes better towards the low frequencies.

The frequency response of the scaling function $\phi$ can be interpreted as the impulse response of a lowpass filter. The companion scaling function of $\mathrm{db} 2$ wavelet function is shown in Figure 13 alongside its Fourier transforms for various normalized scales. It clearly shows a lowpass characteristics but the bandwidth and cut-off frequency reduce as scale $a$ approaches infinity.

These frequency responses demonstrate the different role played by wavelet function at extracting high-frequency information and that of scaling function at deriving lowfrequency information. Appendix B describes the implementation of DWT through successive steps of highpass and lowpass filtering with filters constructed from the wavelet and scaling functions, respectively. Normally, multiple wavelet functions would be used to separate high frequencies into multiple bands while a single scaling function is used to represent the remaining low-frequency component. The highfrequency bands are considered as the detailed information while the low-frequency band is taken as the approximate information. 


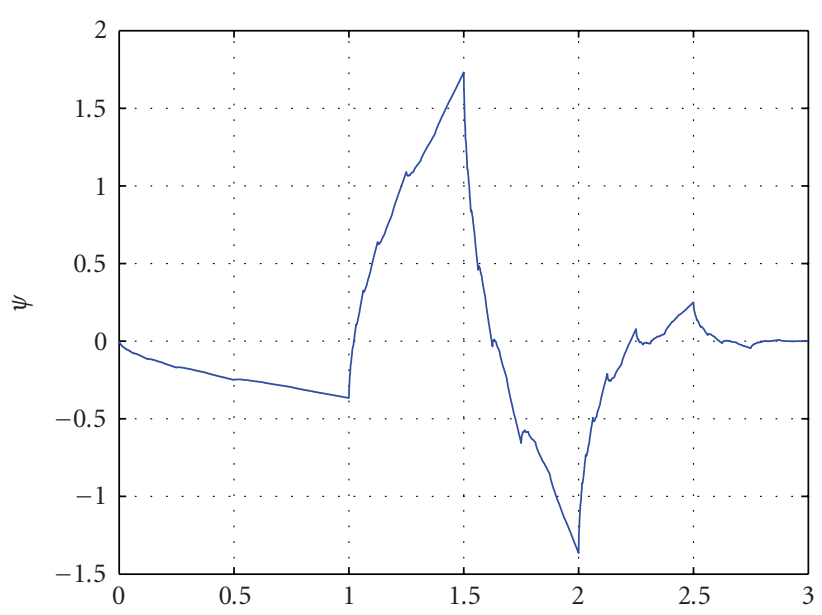

(a)

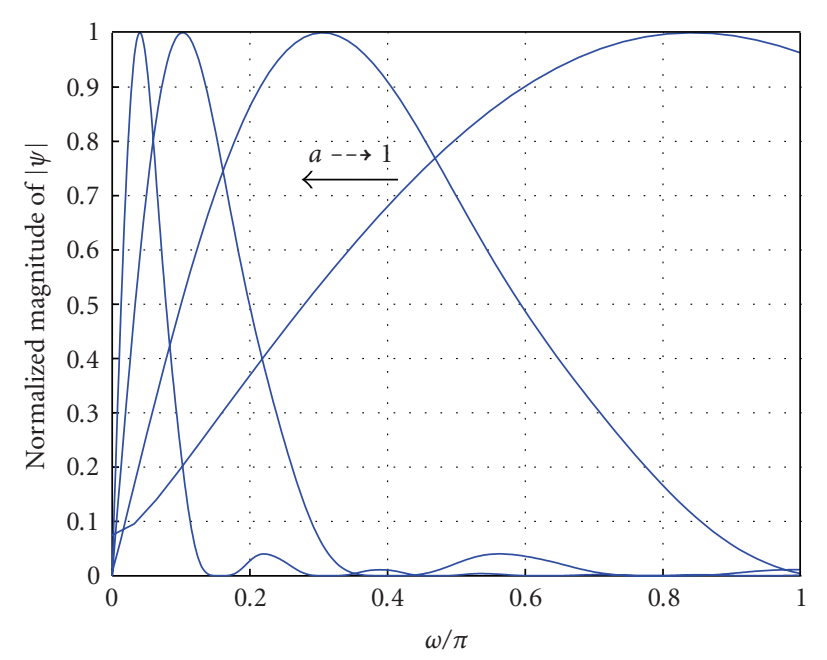

(b)

FIgURE 12: (a) db2 mother wavelet and (b) its Fourier transforms at various scales.

\section{A.2. Fast implementation of DWT}

Recognizing the above frequency responses, the dyadic DWT decomposition is often implemented as a cascaded highpass and lowpass filtering with downsampling as shown in Figure 14. The highpass filter $h[n]$ is determined from both the wavelet and scaling functions, while the lowpass filter $g[n]$ is determined from the scaling function only

$$
\begin{aligned}
& h[n]=\left\langle\frac{1}{\sqrt{2}} \psi\left(\frac{t}{2}\right), \phi(t-n)\right\rangle, \\
& g[n]=\left\langle\frac{1}{\sqrt{2}} \phi\left(\frac{t}{2}\right), \phi(t-n)\right\rangle .
\end{aligned}
$$

The highpass filtering produces the details $D_{j}$ (high frequency) of the decomposition, while the lowpass filtering produces the approximations $A_{j}$ (low frequency). First, the original signal is passed through the two filters producing the detail $D_{1}$ and approximate $A_{1}$ coefficients for $j=1$ (scale $\left.a=2^{1}\right)$. After downsampling by a factor of 2 , the approxi-

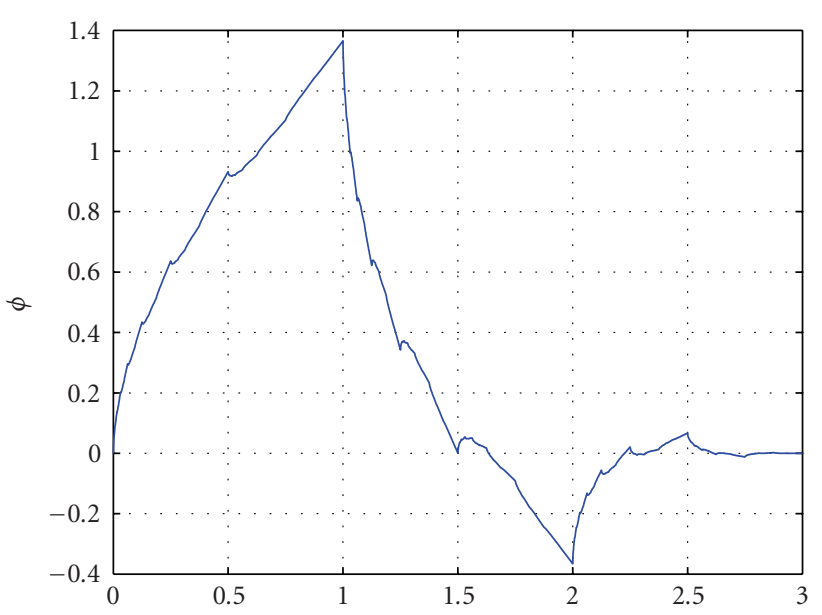

(a)

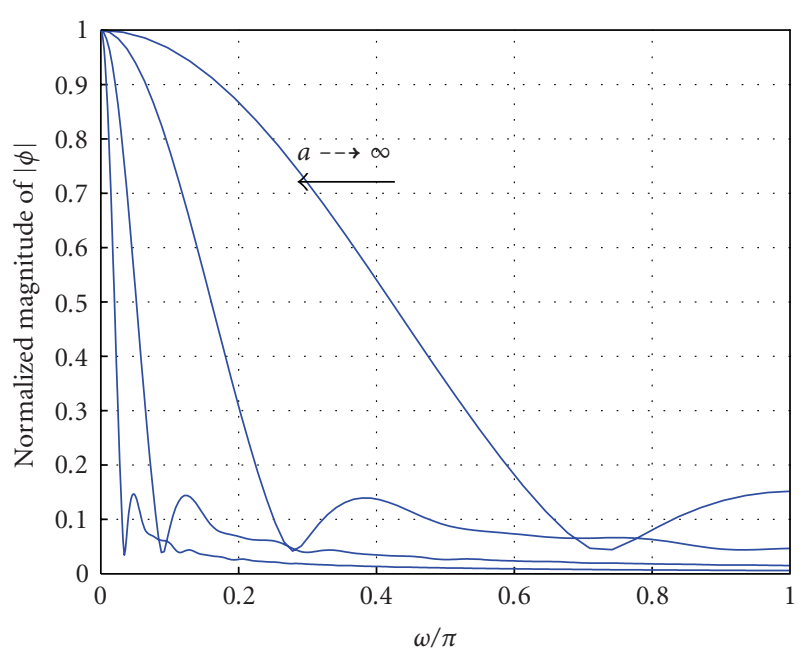

(b)

Figure 13: (a) Scaling function of $\mathrm{db} 2$ and (b) its Fourier transforms at various scales.

mate coefficients $A_{1}$ are passed through the same filters again to obtain the coefficients for $j=2$ (scale $a=2^{2}$ ). After another downsampling, the approximate coefficients $A_{2}$ are then filtered again to obtain the next level of coefficients. This filtering operation can be taken as successive segregations of the same function $f$, with each segregation providing incremental information for a particular frequency band.

In this way, a given signal $f(t)$ is expanded in terms of its orthogonal basis of scaling and wavelet functions. In essence, it is represented by one set of scaling coefficients, and one or several sets of wavelet coefficients

$$
\begin{aligned}
f(t)= & \sum_{k} A_{1}(k) \phi(t-k)+\sum_{k} \sum_{j=1} D_{j}(k) 2^{-j / 2} \psi\left(2^{-j} t-k\right) \\
= & \sum_{k} A_{j_{0}}(k) 2^{-j_{0} / 2} \phi\left(2^{-j_{0}} t-k\right) \\
& +\sum_{k} \sum_{j \leq j_{0}} D_{j}(k) 2^{-j / 2} \psi\left(2^{-j} t-k\right)
\end{aligned}
$$




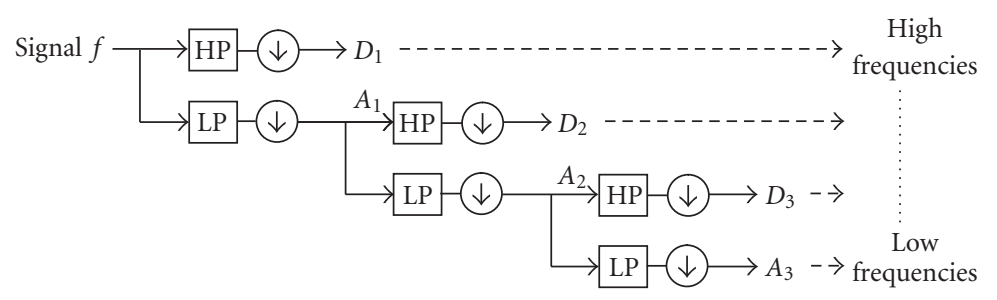

\begin{tabular}{l|l}
\hline HP & Highpass filter \\
\cline { 1 - 1 } LP & Lowpass filter \\
(v) Down-sampling
\end{tabular}

FIgURE 14: Fast DWT decomposition.

where $A_{1}$ is the scaling coefficient as computed from (A.2) while $D_{j}$ is the $j$-level wavelet coefficients computed from (A.1). $k$ denotes the translation in time and scales $j$ denote the different frequency bands, from high to low frequencies for $j=1,2, \ldots$. They can be computed via DWT filters of (A.3) and (A.4) as follows:

$$
\begin{aligned}
& A_{j+1}(k)=\sum_{n} h(n-2 k) A_{j}(n), \\
& D_{j+1}(k)=\sum_{n} g(n-2 k) A_{j}(n) .
\end{aligned}
$$

\section{B. FAMILIES OF WAVELET FUNCTIONS}

One reason for the popularity of wavelet technique is the vast choices of wavelets as the basis function, compared to the fixed choice of sine and cosine functions for Fourier analysis. This enables wavelet analysis to be adapted according to the expected characteristics of the signal. A coarse (irregular) wavelet is more suitable for signal with sharp or abrupt transitions, while those smooth ones are better analyzed using regular wavelets. Figure 15 shows some commonly used wavelet functions and their associated scaling functions plotted on their immediate left. Each function possesses own unique properties that lend them to be more appropriate for certain range of applications. Some of the essential properties are listed and explained below.

(i) Support size: the speed of convergence to zero as time or the frequency goes to infinity, which quantifies both time and frequency localisations.

(ii) Symmetry: useful in avoiding dephasing in image processing.

(iii) Number of vanishing moments: useful for compression purposes as it separates smooth and transient parts of the signal.

(iv) Regularity: useful for getting nice features, like smoothness of the reconstructed signal or image, and for the estimated function in nonlinear regression analysis. (v) Presence of scaling function: needed for representing the low frequencies.

The effectiveness of CWT and DWT is influenced by the choice of mother wavelet and its scaling function, if it exists. Different types of mother wavelets have different properties. There are many wavelet functions and they can be divided into three types according to their orthogonality, namely, redundant wavelets, orthogonal wavelets, and biorthogonal wavelets. Orthogonal and biorthogonal wavelets analysis lead to no redundancy. In addition, biorthogonal wavelets have an added advantage as their filters are symmetric and can be used to reconstruct the original signal. However, not all the properties are significant for power quality applications. The following sections describe several important properties that need to be considered when choosing wavelet functions for power quality applications, which a summary of several popular wavelet functions is given in Table 6 .

\section{B.1. Number of vanishing moments}

A wavelet $\psi$ is defined to have $p$ vanishing moments if

$$
\int_{-\infty}^{+\infty} t^{k} \psi(t) d t=0 \quad \text { for } 0 \leq k<p
$$

where $p$ and $k$ are integers. This means that $\psi$ is orthogonal to any $p-1$ degree polynomial. Therefore, if a signal has regular part whose degree of order is less than $p-1$, the regular part can be readily suppressed during wavelet transform. For a signal $f(t)$ that is continuously differentiable at point zero, it can be expanded into the following Taylor expansion series:

$f(t)$

$$
=\left[f(0)+t f^{\prime(0)}+t^{2} f^{(2)}(0)+\cdots+t^{p-1} f^{(p-1)}(0)\right]+g(t),
$$

where $g(t)$ is the irregular part of the signal $f(t)$. As $p-1$ degree polynomial would be suppressed by wavelet transform, both $g(t)$ and $f(t)$ would have the same detail coefficients.

This means that with sufficient number of vanishing moments, wavelet transform would systematically suppress the 

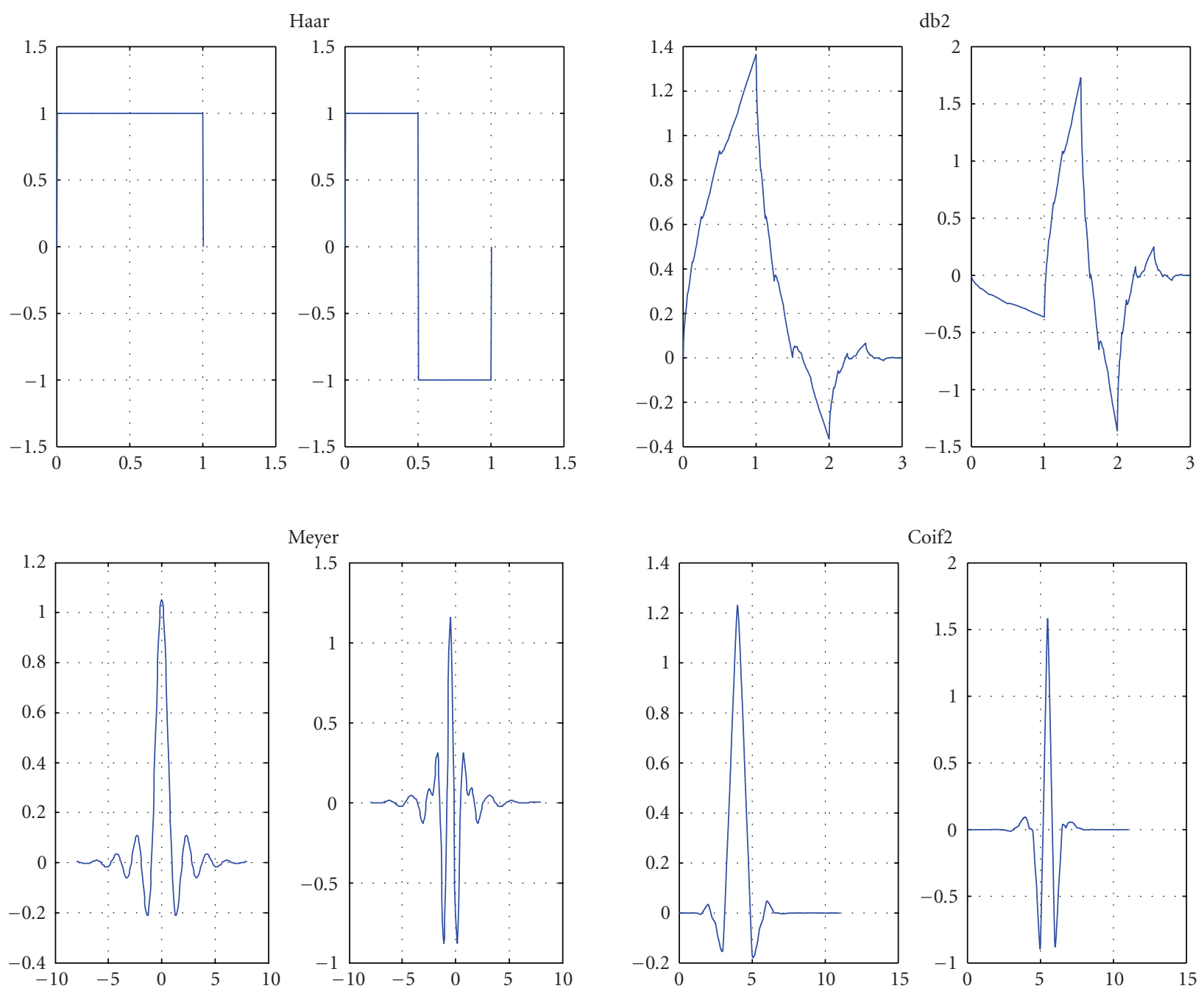

Coif2

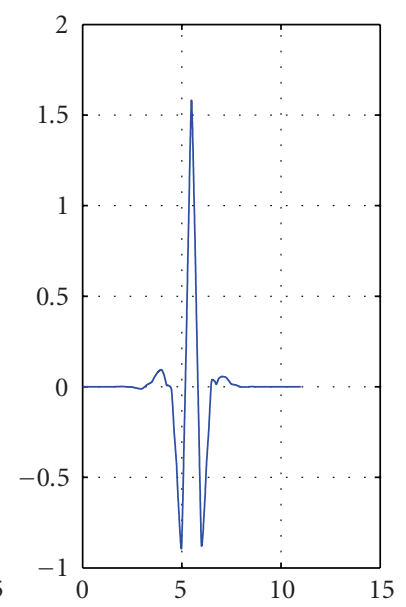

FIGURE 15: Some popular wavelet functions and respective scaling functions.

regular parts of a signal and would focus on the irregular parts $[9,25,26]$. This would help to reveal the irregular parts and is very useful when processing transient signals.

\section{B.2. Support size}

Support size quantifies the localization in time and frequency. If the analyzed signal has a nondifferentiable point that falls within the support of a wavelet function, there may be many high wavelet coefficients. Hence, to keep the number of nonzero coefficients low, it is normally advisable to keep the support size small. However, the wavelet functions used are often orthogonal wavelets, whose support size always increases or decreases in tandem with the number of vanishing moments. For an orthogonal wavelet having $p$ vanishing moments, its support size is at least $2 p-1$ $[9,27]$. As a larger number of vanishing moments are generally preferred for signal suppression purpose, there needs to be a trade-off between the number of vanishing moments and the support size. Although it helps to identify transients or abrupt changes, the subsequent large support size would result in many high coefficients. In general, if a signal is highly irregular, it would be better to decrease the support size at the cost of reducing the number of vanishing moments.

\section{B.3. Regularity}

The regularity of wavelet function has mostly a cosmetic influence on the error introduced by thresholding of the wavelet coefficients. If the function is $r$-time continuously differentiable ( $r$ is a nonnegative integer), then its regularity is $r$. A large $r$ implies a more regular function. For certain wavelet functions such as the Daubechies wavelets, the regularity is linked to the support size. The longer the support a Daubechies wavelet has, the more regular the function is. Regularity is useful in estimating the local properties of a function or signal. With a regular mother wavelet, the 
TABLE 6: Summary of wavelet families.

\begin{tabular}{|c|c|c|c|c|c|c|c|c|c|}
\hline Properties & $\begin{array}{l}\text { Morlet } \\
(\text { morl })\end{array}$ & $\begin{array}{l}\text { Mexican-hat } \\
\text { (mexh) }\end{array}$ & $\begin{array}{l}\text { Meyer } \\
\text { (meyr) }\end{array}$ & $\begin{array}{l}\text { Haar } \\
\text { (Haar) }\end{array}$ & $\begin{array}{l}\text { Daubechies } \\
(\mathrm{dbN})\end{array}$ & $\begin{array}{l}\text { Gaussian } \\
\text { (gaus) }\end{array}$ & $\begin{array}{l}\text { Symlet } \\
(\text { symN) }\end{array}$ & $\begin{array}{l}\text { Coiflet } \\
\text { (coifN) }\end{array}$ & $\begin{array}{l}\text { Biorthogonal } \\
\text { wavelet pairs } \\
\text { (biorNr.Nd) }\end{array}$ \\
\hline Regularity & Infinitely & Infinitely & Infinitely & No & Arbitrary & Infinitely & Arbitrary & Arbitrary & Arbitrary \\
\hline $\begin{array}{l}\text { Compactly } \\
\text { supported } \\
\text { orthogonal }\end{array}$ & No & No & No & 2 & $2 \mathrm{~N}-1$ & No & $2 \mathrm{~N}-1$ & $6 \mathrm{~N}-1$ & $2^{\mathrm{Nd}}+1$ \\
\hline Symmetry & Yes & Yes & Yes & Yes & Asymmetry & Yes & $\begin{array}{l}\text { Near } \\
\text { symmetry }\end{array}$ & $\begin{array}{l}\text { Near } \\
\text { symmetry }\end{array}$ & Yes \\
\hline $\begin{array}{l}\text { Number of } \\
\text { vanishing moments }\end{array}$ & - & - & - & 1 & $\mathrm{~N}$ & - & $\mathrm{N}$ & $2 \mathrm{~N}$ & $\mathrm{Nr}-1$ \\
\hline $\begin{array}{l}\text { Existence of scaling } \\
\text { function }\end{array}$ & No & No & Yes & Yes & Yes & No & Yes & Yes & Yes \\
\hline Orthogonal analysis & No & No & Yes & Yes & Yes & No & Yes & Yes & No \\
\hline Biorthogonal analysis & No & No & Yes & Yes & Yes & No & Yes & Yes & Yes \\
\hline FIR filters (length) & No & No & No & 2 & $2 \mathrm{~N}$ & No & $2 \mathrm{~N}$ & $6 \mathrm{~N}$ & Yes \\
\hline Fast algorithm & No & No & No & Yes & Yes & No & Yes & Yes & Yes \\
\hline CWT & Yes & Yes & Yes & Yes & Yes & Yes & Yes & Yes & Yes \\
\hline DWT & No & No & Yes & Yes & Yes & No & Yes & Yes & Yes \\
\hline Explicit expression & Yes & Yes & No & Yes & No & Yes & No & No & For splines \\
\hline
\end{tabular}

reconstructed signal is smooth. It is important to take note of this property if reconstruction is necessary.

\section{B.4. Symmetry}

Symmetric wavelets show no preferred direction or emphasis in time, while asymmetric wavelets give unequal weighting to different directions. If a wavelet function is symmetric, it is easier to deal with the boundaries of the signal, because the phase shift caused by this wavelet function is linear. Compared to nonlinear phase shift, linear phase shift is generally more acceptable, especially in image processing. If the mother wavelet is not symmetric, the wavelet transform of the mirror of an image is not the mirror of the image's wavelet transform. In power quality assessment, the influence of the signal border distortion of the signal can be mitigated by using symmetric mother wavelets [28].

\section{B.5. Choosing mother wavelet}

To choose a suitable mother wavelet, the properties of the wavelet function must be considered carefully. Generally, the objective of the wavelet analysis and the characteristic of the analyzed object decide the mother wavelet. If a signal has a few isolated singularities and is very regular between the singularities, a wavelet with many vanishing moments can be chosen to produce mainly small coefficients. If there are many singularities, it would be better to decrease the support size at the cost of lower number of vanishing moments. Wavelet analysis that overlaps the singularities of a signal would create high coefficients. Furthermore, if signal reconstruction is needed, a more regular wavelet is preferred in order to obtain a smoother reconstructed signal. Finally, if the analysis is to be carried out on some specific frequencies, the center-frequency of the wavelet, the sampling frequency of the signal, and the length of the signal must be taken into consideration simultaneously. The selection can be done in two steps [29].

Step 1 (Determine the wavelet type). If a fast algorithm is needed, the wavelet must be achievable via FIR filters. If the approximation part of the analysis is needed, scaling function becomes necessary. If reconstruction is needed, biorthogonal wavelets have some advantages compared to orthogonal wavelets. The symmetry and exact reconstruction are both possible with biorthogonal wavelet functions. These requirements help to narrow the selection to one of the following four categories.

\section{Orthogonal wavelets with FIR filters}

These wavelets can be defined through a scaling filter. Predefined families of such wavelets include Haar, Daubechies, Coiflet, and Symlet.

\section{Biorthogonal wavelets with FIR filters}

These wavelets can be defined through two scaling filters for reconstruction and decomposition, respectively. The BiorSplines wavelet family is a predefined family of this category. 


\section{Orthogonal wavelets without FIR filter, but with scaling function}

These wavelets can be defined through the definition of a wavelet function and a scaling function. The Meyer wavelet family is a predefined family of this category.

\section{Wavelets without FIR filter and without scaling function}

These wavelets can be defined through the definition of a wavelet function only. Predefined families of such wavelets include Morlet and Mexican_hat.

Step 2 (Define the orders of wavelet within a selected family). Some wavelet families have a single wavelet function such as Haar, Meyer, and Morlet, while some have many such as Daubechies and Coiflet. If the selected wavelet function is from the latter group, the order of the selected wavelet function must be decided. This involves looking at their properties including the number of vanishing moments, regularity and support size.

In addition, not all wavelet functions have a scaling function equivalent. Hence, only those with scaling function can represent low-frequency information in the wavelet domain. The others would have only high-frequency representation. In addition, many wavelet functions like the Daubechies family of wavelets do not have explicit expression. Hence, it is necessary to construct the wavelet and scaling functions in order to evaluate their performance.

\section{REFERENCES}

[1] IEC 61000-2-1, "Electromagnetic compatibility (EMC)—part 2: environment - section 1: description of the environment - electromagnetic environment for low-frequency conducted disturbances and signalling in public power supply systems," 1st edition, 1990.

[2] IEEE Std. 1159-1995, "IEEE Recommended Practice for Monitoring Electric Power Quality," 1995.

[3] IEC 61000-2-2, "Electromagnetic compatibility (EMC)—part 2-2: environment - compatibility levels for low-frequency conducted disturbances and signalling in public low-voltage power supply systems," 2nd edition, 2002.

[4] I. Y.-H. Gu and E. Styvaktakis, "Bridge the gap: signal processing for power quality applications," Electric Power Systems Research, vol. 66, no. 1, pp. 83-96, 2003.

[5] I. Y.-H. Gu and M. H. J. Bollen, "Time-frequency and timescale domain analysis of voltage disturbances," IEEE Transactions on Power Delivery, vol. 15, no. 4, pp. 1279-1284, 2000.

[6] A. Bruce, D. Donoho, and H.-Y. Gao, "Wavelet analysis [for signal processing]," IEEE Spectrum, vol. 33, no. 10, pp. 26-35, 1996.

[7] A. Graps, "An introduction to wavelets," IEEE Computational Science \& Engineering, vol. 2, no. 2, pp. 50-61, 1995.

[8] R. M. C. Fernández and H. N. D. Rojas, "An overview of wavelet transforms in power system," in Proceedings of the 14th Power System Computational Conference (PSCC '02), Sevilla, Spain, June 2002.

[9] S. Mallat, A Wavelet Tour of Signal Processing, Academic Press, San Diego, Calif, USA, 2nd edition, 1999.
[10] D. C. Robertson, O. I. Camps, J. S. Mayer, and W. B. Gish Sr., "Wavelets and electromagnetic power system transients," IEEE Transactions on Power Delivery, vol. 11, no. 2, pp. 1050-1056, 1996.

[11] M. Karimi, H. Mokhtari, and M. R. Iravani, "Wavelet based on-line disturbance detection for power quality applications," IEEE Transactions on Power Delivery, vol. 15, no. 4, pp. 12121220, 2000.

[12] W. A. Wilkinson and M. D. Cox, "Discrete wavelet analysis of power system transients," IEEE Transactions on Power Systems, vol. 11, no. 4, pp. 2038-2044, 1996.

[13] K. L. Butler-Purry and M. Bagriyanik, "Characterization of transients in transformers using discrete wavelet transforms," IEEE Transactions on Power Systems, vol. 18, no. 2, pp. 648656, 2003.

[14] O. Poisson, P. Rioual, and M. Meunier, "Detection and measurement of power quality disturbances using wavelet transform," IEEE Transactions on Power Delivery, vol. 15, no. 3, pp. 1039-1044, 2000.

[15] S. Santoso, W. M. Grady, E. J. Powers, J. Lamoree, and S. C. Bhatt, "Characterization of distribution power quality events with Fourier and wavelet transforms," IEEE Transactions on Power Delivery, vol. 15, no. 1, pp. 247-254, 2000.

[16] Y.-Y. Hong and C.-W. Wang, "Switching detection/classification using discrete wavelet transform and self-organizing mapping network," IEEE Transactions on Power Delivery, vol. 20, no. 2, part 2, pp. 1662-1668, 2005.

[17] T. X. Zhu, S. K. Tso, and K. L. Lo, "Wavelet-based fuzzy reasoning approach to power-quality disturbance recognition," IEEE Transactions on Power Delivery, vol. 19, no. 4, pp. 1928-1935, 2004.

[18] Z.-L. Gaing, "Wavelet-based neural network for power disturbance recognition and classification," IEEE Transactions on Power Delivery, vol. 19, no. 4, pp. 1560-1568, 2004.

[19] C.-H. Lin and M.-C. Tsao, "Power quality detection with classification enhancible wavelet-probabilistic network in a power system," IEE Proceedings: Generation, Transmission and Distribution, vol. 152, no. 6, pp. 969-976, 2005.

[20] IEC 61000-4-7, "Electromagnetic compatibility (EMC)—part 4-7: testing and measurement techniques - general guide on harmonics and interharmonics measurement and instrumentation, for power supply systems and equipment connected thereto," International Electrotechnical Commission, 2002.

[21] J. Barros and R. I. Diego, "Application of the wavelet-packet transform to the estimation of harmonic groups in current and voltage waveforms," IEEE Transactions on Power Delivery, vol. 21, no. 1, pp. 533-535, 2006.

[22] M. M. Saied, "Capacitor switching transients: analysis and proposed technique for identifying capacitor size and location," IEEE Transactions on Power Delivery, vol. 19, no. 2, pp. 759-765, 2004.

[23] H. P. William, P. F. Brian, A. T. Saul, and T. V. William, Numerical Recipes in C: The Art of Scientific Computing, Cambridge University Press, Cambridge, UK, 2nd edition, 1992.

[24] D. W. Durbak, "Modeling guidelines for switching transients, modeling and analysis of system transients," IEEE PES special publication, 1998.

[25] W.-L. Hwang and S. Mallat, "Singularities and noise discrimination with wavelets," in Proceedings of IEEE International Conference on Acoustics, Speech, and Signal Processing (ICASSP '92), vol. 4, pp. 377-380, San Francisco, Calif, USA, March 1992. 
[26] S. Mallat and W.-L. Hwang, "Singularity detection and processing with wavelets," IEEE Transactions on Information Theory, vol. 38, no. 2, part 2, pp. 617-643, 1992.

[27] I. Daubechies, Ten Lectures on Wavelets, SIAM, Philadelphia, Pa, USA, 1992.

[28] S.-J. Huang, C.-T. Hsieh, and C.-L. Huang, "Application of Morlet wavelets to supervise power system disturbances," IEEE Transactions on Power Delivery, vol. 14, no. 1, pp. 235-243, 1999.

[29] User's Guide of Wavelet Toolbox, MathWorks, 2004, http:// www.mathworks.com/.

S. Chen received his B.E. (Honors) and Ph.D. degrees from the University of Canterbury, Christchurch, New Zealand, in 1992 and 1996, respectively. He has worked as Postdoctoral Fellow with the same university on power-quality-related projects. $\mathrm{He}$ is now an Associate Professor in the School of Electrical and Electronic Engineering, Nanyang Technological University, Singapore. His research interests include in-

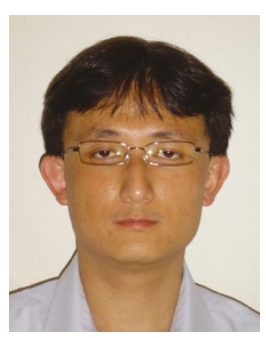
formation theory with applications in power quality monitoring, analysis and assessment, computer modeling and simulation of power systems, power market and power line communications.

H. Y. Zhu received her B.E., M.Eng., and Ph.D. degrees from the Huazhong University of Science and Technology, China, in 1996 and 1999, Nanyang Technological University, Singapore, in 2006, respectively. She was a Protection Device Design Engineer of Wuhan Guoce Electrical New Technological Co. Ltd., China, from 1999 to 2001. Her research interests include power system protection and signal processing ap-

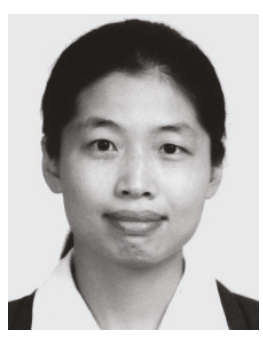
plications in power quality. 